\title{
PCDH7 Promotes Cell Migration by Regulating Myosin Activity
}

\author{
Mohammad Haroon Qureshi, ${ }^{1,2}$, M. Talha Cinko ${ }^{1}$, Halil Bayraktar ${ }^{3}$, Cansu Akkaya ${ }^{1}$, Altug \\ Kamacioglu$^{1}$, Z. Cansu Uretmen-Kagiali ${ }^{1}$, Erdem Bozluolcay ${ }^{1}$, Nurhan Ozlu ${ }^{1,4 *}$ \\ ${ }^{1}$ Department of Molecular Biology and Genetics, Koç University, 34450, Istanbul, Turkey \\ 2 Biomedical Sciences and Engineering Graduate Program, Koç University, 34450, Istanbul, \\ Turkey \\ ${ }^{3}$ Molecular Biology and Genetics Department, Istanbul Technical University, 34469, Istanbul, \\ Turkey \\ ${ }^{4}$ Koç University Research Center for Translational Medicine (KUTTAM), 34450, Istanbul, \\ Turkey
}

*Correspondence and requests for materials should be addressed to NO (email: nozlu@ku.edu.tr) 


\begin{abstract}
Cell migration requires spatiotemporally coordinated activities of multicomponent structures including the actomyosin cortex, plasma membrane, adhesion complexes and the polarity proteins. How they function together to drive this complex dynamic process remains an outstanding question. Here, we show that a member of the protocadherin family, PCDH7 displays a polarized localization in migratory cells with a dynamic enrichment at the leading and rear edges. Perturbation of PCDH7 interferes with the migration of nontransformed retinal pigment epithelial cells and invasion of cancer cells. The overexpression of PCDH7 enhances the migration capability of cortical neurons in vivo. PCDH7 interacts with the myosin phosphatase subunits MYPT1 and PP1c $\beta$ and it enhances the phosphorylation of regulatory light chain and ERM at the leading and rear edges of migratory cells. The chemical inhibition of phosphatase activity recovers migration phenotypes of PCDH7 knockout cells. We propose that $\mathrm{PCDH} 7$ regulate phosphorylation thus activity of myosin and ERM at the polarized cortex by quenching myosin phosphatase that results in a higher persistence of migrating cells. Collectively, our study suggests a new mechanism for the spatial coordination of plasma membrane and the cortex during cell migration.
\end{abstract}

Keywords: cell cortex, myosin, protocadherin, cell migration, ERM 


\section{Introduction}

Cell motility is an indispensable feature of a wide range of biological processes including mammalian embryonic development, inflammatory response, and tissue repair and regeneration (Ridley et al., 2003; Te Boekhorst et al., 2016). Cell's motility also features in the invasion and metastasis of tumor cells (Hanahan and Weinberg, 2011; Schwartz and Horwitz, 2006; Svitkina, 2018; Te Boekhorst et al., 2016). During cell migration, actin-based structures, lamellipodia and filopodia forms at the leading edge of migrating cells (Cramer, 1999; Cramer, 2013; Cramer et al., 1997). Protrusive forces are generated by those structures through polymerization of actin filaments (Svitkina, 2018) which is regulated by ERM (Ezrin-Radixin-Moesin) proteins (Arpin et al., 2011). Phosphorylation of ERM by various kinases stimulates lamellipodium and filopodium formation thus membrane protrusion and cortical stability through its simultaneous interaction with F-actin and plasma membrane (Baumgartner et al., 2006; Hipfner et al., 2004; Ponti et al., 2004; Uretmen Kagiali et al., 2020).

The contractility of the actomyosin cytoskeleton is mediated by the motor activity of myosins, mainly myosin II, also known as the non-muscle myosin II (NMII), which plays a central role in cell migration (Vicente-Manzanares et al., 2009). The Myosin II-based force generation is dynamically controlled through the phosphorylation of its light chain, Myosin Light Chain 2 (MLC2)/ Regulatory Light Chain (RLC). Phosphorylation of MLC2 at Serine19 (S19) reversibly drives the ATPase activity of myosin II by enhancing the $\mathrm{Mg}^{2+}$-ATPase activity of the complex in the presence of actin (Houdusse and Sweeney, 2016; Somlyo and Somlyo, 2003). A second phosphorylation of MLC2 at Threonine18 (T18) further enhances the $\mathrm{Mg}^{2+}$-ATPase activity of myosin II (Ikebe et al., 1986). Phosphorylation of MLC2 triggers myosin's binding to actin and 
can be governed by the activity of more than a dozen kinases including MLCK, ROCK, ZIPK, CDC42BP (Vicente-Manzanares et al., 2009). Similarly, MLC2 dephosphorylation by phosphatases such as myosin phosphatase also regulates the motor activity of the myosin complex (Ito et al., 2004; Kiss et al., 2019). Myosin motors-based contractility plays a central role in all steps of cell migration through its interaction with actin cytoskeleton. Although its impact in retraction, protrusion and adhesion of a migratory cell is well known (Cai et al., 2006; JuanesGarcia et al., 2015; Schwartz and Horwitz, 2006; Vicente-Manzanares et al., 2008; VicenteManzanares et al., 2009; Vicente-Manzanares et al., 2007), the regulation of the complex interplay between myosin and other cortical and plasma membrane elements is incompletely understood.

Members of the cadherin superfamily exhibit differential roles in the development and progression of cancer (Jeanes et al., 2008; Mendonsa et al., 2018; Mrozik et al., 2018). Members of the largest subfamily of the cadherin superfamily, namely protocadherins, are widely expressed in the nervous system. Their functions are poorly explored but they are gradually being shown to play important roles in cell-cell interactions in the nervous system (Hayashi and Takeichi, 2015). Non-clustered protocadherins, a subset of protocadherins, have recently been suggested to play important roles in neuronal functions and cell migration. Unlike the classical cadherins, their cell adhesion role is context dependent, as they can also exhibit anti-adhesion roles during development and cell division (Jontes, 2016; Ozlu et al., 2015; Qiu et al., 2016; Yasuda et al., 2007; Zhang et al., 2012; Zhou et al., 2017; Zhu et al., 2014). One such member, PCDH7, belongs to the $\delta 1$ subclass of nonclustered protocadherins. Its roles in mammalian cells are not well characterized. PCDH7 has been reported to be highly expressed in brain metastasizing breast cancer cell lines (Bos et al., 2009). PCDH7 mediated metastasis of breast cancer cells to brain is primarily driven by cell-cell contacts 
between astrocytes and invading cancer cells. PCDH7 stabilizes connexin-43 mediated gap junctions and drives STING signaling across the cells, thus enhances the survival of invading cells (Chen et al., 2016). A Xenopus homolog of PCDH7, NF-protocadherin (NF-PC) has been previously shown to play a role in ectoderm differentiation and retinal axon growth and guidance through its interaction with TAF1/SET (Heggem and Bradley, 2003; Leung et al., 2015; Leung et al., 2013; Piper et al., 2008). TAF1/SET is an inhibitor of phosphatase, PP2A. A recent work showed that PCDH7 mediates tumor growth by inducing MAPK signaling through SET mediated PP2A inhibition (Zhou et al., 2017).

Our previous work has shown that PCDH7 enriches and localizes all over the cell surface during mitosis, but it is largely restricted to cell-cell contact regions in interphase cells (Ozlu et al., 2015). Perturbation of PCDH7 expression affected cell rounding pressure of mitotic cells, an effect also seen upon myosin IIA heavy chain (MYH9) perturbation (Ozlu et al., 2015). Despite emerging role for PCDH7 in cellular cortex, its molecular mechanism in mammalian cells remain to be uncovered. In this study, we explore PCDH7's role in cell migration alongside with cortical actomyosin. Our work shows that PCDH7 is associated with cortical actomyosin with a dynamic enrichment at the leading and rear edges. RPE1 cells lose their persistence of migration upon PCDH7 perturbation, an effect that is recovered upon PCDH7 replenishment. In this study for the first time, we showed that PCDH7 interacts with the myosin phosphatase subunits PP1c $\beta$ and MYPT1 and spatially controls its dephosphorylation activity. Thereby, PCDH7 maintains a focal pool of phosphorylated myosin light chain 2 (MLC2) and ERM at the leading edge enabling phosphorylated myosin complexes and ERM to establish mechanical properties of polarized cortex and trigger persistent migration. 


\section{Results}

PCDH7 displays dynamic and polarized cell surface enrichment at the leading and rear edges in migrating RPE1 cells

Because of their distinct polarized morphology and migratory behavior, we used Retinal Pigment Epithelial cells (RPE1) which are non-transformed adherent human cells to characterize the role of PCDH7 during migration. Classical cadherins and specific protocadherins are known to be connected to the cortical actomyosin cytoskeleton (Budnar and Yap, 2013; Hayashi et al., 2014; Moeller et al., 2004; Ramakrishnan et al., 2012; Ratheesh and Yap, 2012). PCDH7 has four isoforms, among them isoform $\mathrm{a}$ and isoform $\mathrm{b}$ are shorter and isoform $\mathrm{c}$ and isoform $\mathrm{d}$ are the longer versions. To investigate the dynamics of $\mathrm{PCDH} 7$ and F-actin proteins during migration, the longer isoform PCDH7c-GFP and lifeact-RFP fusion proteins expressing RPE1 cells were imaged using time-lapse confocal scanning microscopy. A non-polarized cell was initially chosen for imaging to monitor PCDH7's dynamics as it polarizes. When the cell was in a non-polarized orientation, PCDH7c-GFP displayed a uniform cell surface distribution (Video 1, Figure 1A, top panel). However, the localization of PCDH7-GFP changed as the cell polarized and started to migrate (Video 1, Figure 1A, middle and lower panel). PCDH7c was enriched at the protruding leading edge and trailing rear edge (Video 1, Figure 1A). PCDH7 also prominently decorated the retraction fibers which are a network of long and thin traces rich in actin at the trailing rear edge (Figure 1A, lower panel) (Cramer et al., 1997). In polarized cells, PCDH7 enriched at the leading edge where it largely co-localized with F-actin highlighted by lifeact-RFP (Supplementary Figure S1A, Video 2). PCDH7 also displays a dominant vesicular presence inside the cell, owing to its enrichment in the endoplasmic reticulum as shown by our previous study (Ozlu et al., 2015). 


\section{PCDH7's cortical dynamics in migratory cells are dependent on intact cortical actomyosin}

\section{assembly}

During actin-based cell migration, myosin II enriches at the rear edge and moves the cell forward by its motor activity causing rear edge retraction(Conti and Adelstein, 2008; Cramer, 2013; Vicente-Manzanares et al., 2009). Active form of myosin complex displays phosphorylation at MLC2 (S19). We asked whether the polarized surface enrichment of PCDH7 as observed in live imaging is correlated with phosphorylated MLC2, (pMLC2, S19). The colocalization of PCDH7 with pMLC2 was pronounced in both leading (Figure 1B, top) and rear edges (Figure 1B, bottom) This implicates that PCDH7's localization at the leading and rear edges are associated with active forms of myosin II.

Next, we investigated PCDH7's dependency on myosin II activity. Blebbistatin is a wellcharacterized small-molecule inhibitor of myosin's motor activity (Straight et al., 2003). After blebbistatin treatment, both the organization of F-actin and PCDH7 at the cell membrane appeared to be disrupted (Video 3). As the cell shrinks after blebbistatin treatment, PCDH7's polarized leading and rear edge enrichment was perturbed and PCDH7c was evenly distributed across the whole cell surface (Supplementary Figure S1B). We concluded that myosin motor activity is required for PCDH7's polarized localization at the cell surface during cell migration.

\section{PCDH7 depletion affects cell migration and persistence}

To investigate PCDH7's role in cell migration, we used CRISPR/Cas9 method to knock-out

PCDH7 in RPE1 cells. According to the western-blotting analysis, PCDH7 expression is absent in 
PCDH7 knockout (KO) (Figure 2A). In addition to KO, add-back experiments were performed by the ectopic expression of isoform $\mathrm{C}$ of $\mathrm{PCDH} 7(\mathrm{PCDH} 7 \mathrm{c})$ to eliminate the possibility of off-target effects (Figure 2A). To analyze the effect of PCDH7 knockout on random migration, we performed single cell tracking of RPE1 cells. Single cell trajectories of KO cells were remarkably confined as compared to those of non-targeting (NT) control, whereas upon replenishment of PCDH7c $(\mathrm{KO}+\mathrm{PCDH} 7 \mathrm{c})$, cells once again exhibited outreaching longer trajectories (Figure 2B, video 4). In addition, knockout cells displayed a loss of persistence phenotype (Figure 2C and Video 4), which was recovered after the expression of $\mathrm{PCDH} 7 \mathrm{c}$ in knockout cells $(\mathrm{KO}+\mathrm{PCDH} 7 \mathrm{c})$ (Figure $2 \mathrm{C})$. We also calculated angular displacement which is defined as the angular shift between two subsequent trajectories of a single cell. We focused on two sets of angular shifts: small angle shifts of trajectories $\left(0^{0}-36^{0}\right)$ and large angle shifts of trajectories $\left(144^{0}-180^{0}\right)$. A higher proportion of small angle changes $\left(0^{0}-36^{0}\right)$ would indicate a stronger decisive behavior to migrate in the direction of initial migration, whereas large angular changes $\left(144^{0}-180^{\circ}\right)$ would indicate a lesser decisiveness and more back and forth futile attempts and thus, lesser persistence. Our analysis of angular displacements indicated a reduction in the proportion of the small angle changes between NT and $\mathrm{KO}$ sets (Figure 2D). Upon recovery of $\mathrm{PCDH7}$ expression $(\mathrm{KO}+\mathrm{PCDH} 7 \mathrm{c})$, the proportion of small angle changes was increased (Figure 2D). On the other hand, analysis of large angular shifts $\left(144^{0}-180^{\circ}\right)$ showed a significant increase of these angular shifts in KO cells. This increase, however, is reduced in $\mathrm{KO}+\mathrm{PCDH} 7 \mathrm{c}$ cells (Figure 2E). We also used the shorter isoform $\mathrm{PCDH} 7 \mathrm{~b}$ for assessing the recovery upon knockout and observed a significant recovery in persistence (Figure 2F). In parallel, we assessed the migration phenotype by performing shRNA mediated knockdown of PCDH7 in RPE cells. Three different shRNAs against PCDH7 significantly reduced its expression (Supplementary Figure S2A). Since shRNA1 and shRNA2 showed a similar level 
of reduction in PCDH7 expression, we decided to use shRNA2 and shRNA3 for the phenotype assessment. In line with the knockout results, shRNA mediated depletion of PCDH7 significantly diminished RPE cell migration capacity as evident through multiple parameters (Figure $2 \mathrm{G}$ and Supplementary Figure S2B-D). Plots of single cell trajectories revealed that PCDH7 silenced cells traveled shorter distance than non-silencing (NS) cells (Supplementary Figure S2B). Upon PCDH7 depletion, individual migration trajectories of cells displayed reduced persistence of migration as compared to the NS set (Figure 2G). Similarly, angular displacement profiles upon PCDH7 knockdown were like that of PCDH7 knockout (Supplementary Figure S2C-D), i.e. upon PCDH7 knockdown, small angle displacement proportion decreased while large angular displacement proportion increased. Collectively, our data suggested that $\mathrm{PCDH7}$ is required for persistent directional cell migration, and cells lose their directionality and thus, lose their persistent migration ability upon PCDH7 perturbation.

To further check the effects of PCDH7 in the context of cancer cells, we tested for migration defects in HeLaS3 cells. Two HeLaS3 PCDH7 knockout cell lines were generated using CRISPR/Cas9 system (Supplementary Figure S2E) and were used to compare their migration ability using xCELLigence Real Time Cell Analysis for cell migration (Figure 2H). Essentially, this assay measures directional migration rather than random migration. Knockout cells displayed a significant reduction in cell migration compared to control cell lines. This result suggests that just like random migration, $\mathrm{PCDH} 7$ perturbation also affects directional migration.

\section{PCDH7 expression correlates with phosphorylation of MLC2}


Polarized enrichment of PCDH7, and its colocalization with actomyosin, prompted us to explore its functional association with myosin II. To test whether PCDH7 plays a role in the activation of myosin II during migration, we biochemically tested pMLC2 levels (S19) in the non-targeting (NT) control and PCDH7 knockout (KO) HeLa S3 cells by Western Blotting. Strikingly, PCDH7 knockout HeLa S3 cells exhibited significantly reduced pMLC2 levels compared to control (Figure 3A). To examine the myosin activation spatially, we performed immunofluorescence for p-MLC2 levels in PCDH7 knockout RPE1 cells. We observed a dramatic reduction in pMLC2 levels at the leading edges in PCDH7 knockout cells, a phenotype that is recovered with both PCDH7 isoforms replenishment (Figure 3B-C).

Next, we performed PCDH7 overexpression in wild type RPE1 cells to observe its effect on MLC2 phosphorylation. Upon overexpression of PCDH7 isoforms, p-MLC2 levels were significantly elevated in the leading edges (Figure 3D-E). Taken together, our results indicated that polarized PCDH7 localization at the leading and rear edges colocalize with pMLC2 and perturbation, recovery and overexpression experiments demonstrate that $\mathrm{PCDH7}$ expression levels regulate MLC2 phosphorylation at the leading edge of polarized cells.

\section{PCDH7 interacts with myosin phosphatase to enhance MLC2 phosphorylation.}

Next, we focused on exploring the molecular mechanism through which PCDH7 mediates MLC2 phosphorylation. To tackle this, we performed pulldown of PCDH7c-GFP fusion protein followed by LC-MS/MS for identification of its interaction partners. Our results revealed components of actomyosin as the most significantly enriched proteins associated with PCDH7, including actin isoforms, subunits of myosin II isoforms (Figure 4A, green box), ERM complex (Figure 4A, blue 
box) and various other actin cytoskeleton regulatory proteins. We observed clusters involved in the regulation of actin cytoskeleton, cadherin binding, and actomyosin regulation, among others (Figure 4A, Supplementary Table S1).

Interestingly, we observed several component subunits of phosphatases such as MYPT1 (PPP1R12A), PP1c $\beta / \delta$ (PPP1CB), PP1c $\gamma$ (PPP1CC), and PPP2R1A, as interaction partners (Figure 4A, red box). Both MYPT1 and PP1c $\beta$ are components of myosin phosphatase which regulates the phosphorylation of MLC2 (Kiss et al., 2019). The component PP1c $\beta$ is the catalytic subunit whereas MYPT1 is the myosin targeting subunit of myosin phosphatase holoenzyme (Shirazi et al., 1994). We performed immunofluorescence for MYPT1 and PCDH7 isoforms. Our results showed colocalization of PCDH7 isoforms with MYPT1 on the cell front (Figure 4B). To further analyze the interaction of PCDH7 and MYPT1, we performed cotransfection of PCDH7V5 along with EGFP-PP1c $\beta$-MYPT1, or EGFP- PP1c $\beta^{*}$ (D63A)-MYPT1 (with catalytically inactive PP1c $\beta$ ). Earlier work has shown a better substrate identification with mutant PP1 subunits $\left(\mathrm{PP} 1 \mathrm{c} \beta^{*}(\mathrm{D} 63 \mathrm{~A})\right)$ wherein substrates are trapped in the complex rather than separating as happens during the wild type PP1 catalytic activity (Szklarczyk et al., 2019). Our results showed the interaction of PCDH7c with both EGFP-PP1c $\beta$-MYPT1, and EGFP-PP1c $\beta *(D 63 \mathrm{~A})-\mathrm{MYPT} 1$ (Figure 4C). Additionally, EGFP-MYPT1 and EGFP-PP1c $\beta$ separately also indicate interactions with PCDH7c (Supplementary Figure S4A).

We also performed Proximity Ligation Assay (PLA) to further ascertain an interaction between PCDH7 and MYPT1. We used an anti-GFP antibody targeting PCDH7c-GFP and an anti-MYPT1 antibody for PLA. Our results indicated an interaction between PCDH7c and MYPT1 when anti- 
GFP and anti-MYPT1 are used together as compared to anti-GFP or anti-MYPT1 alone, further strengthening the evidence of interaction of PCDH7 and MYPT1 (Supplementary Figure S4B, Figure 4D).

In Figure 4A-D, the longer isoform of PCDH7 (PCDH7c) was used. Since we observed that PCDH7b also rescues the cell migration defect (Figure 2F) and restores pMLC2 levels (Figure 3BC), we tested whether PCDH7b also interacts with myosin phosphatase by performing immunoprecipitation. We performed cotransfection of PCDH7b-V5 along with EGFP-PP1c $\beta$-, and EGFP- MYPT1 in HelaS3 cells. Our results indicate that PCDH7b also interacts with myosin phosphatase components (Supplementary Figure S4C-D).

To test the interdependency between MYPT1 and PCDH7 enrichment, we performed immunofluorescence imaging of MYPT1 in PCDH7 knockout cells. We observed a reduction in MYPT1 levels at the leading edges upon PCDH7 KO, and this reduction was recovered upon $\mathrm{PCDH7c}$ and PCDH7b isoforms expression in KO cells (Supplementary Figure S3A-B). We further analyzed the overexpression of PCDH7 in wild type cells and observed that MYPT1 was enriched at the leading edges upon PCDH7c or PCDH7b expression (Supplementary Figure S3CD). Taken together, our results showed that PCDH7, both isoform b and c, interact with myosin phosphatase subunit MYPT1 and PCDH7 expression correlates with MYPT1 enrichment at the leading edge.

PCDH7 facilitates Ezrin Activation at the Leading Edges 
All the components of ERM complex were identified as the interactor of PCDH7 in our mass spectrometry analysis (Figure 4A), which prompted us to investigate the interaction between PCDH7 and the actin binding ERM (ezrin/radixin/moesin) proteins. Confocal live imaging of RPE1 cells and immunofluorescence data showed high correlation between PCDH7-GFP and Ezrin-RFP signals at the cell membrane/cortex (Video 5 and Supplementary Figure S5A). In agreement with these results, when we performed immunoprecipitation using PCDH7-GFP expressing cells, we confirm the PCDH7 and Ezrin interaction (Supplementary Figure 5B). We suggest that PCDH7's interaction with the ERM mediates its association with the actin cytoskeleton at the cell cortex.

The activation of Ezrin protein is triggered by the phosphorylation of its carboxyl-terminal (T567), which stimulates its F-actin binding (Bosk et al., 2011). The active form of Ezrin is crucial for the establishment of leading edge and cell motility (Prag et al., 2007). Given the fact that Myosin Phosphatase regulates ERM proteins by dephosphorylating them (Kiss et al., 2019), we tested whether PCDH7 affects ezrin activation at the leading edges. PCDH7 signal at the cell front show correlation with phospho-ERM (p-ERM) (Supplementary Figure S5C). We compared phosphoERM levels at the leading edge in control, $\mathrm{PCDH7} \mathrm{KO}$ and $\mathrm{PCDH} 7$ recovered cells. Upon PCDH7 knockout, p-ERM levels are reduced at the leading edge and this reduction gets replenished upon PCDH7 isoforms expression (Supplementary Figure S6D).

\section{In vivo radial neuronal migration is enhanced by PCDH7 overexpression}

Protocadherins are highly expressed in neurons and there are increasing reports highlighting that protocadherins play diverse roles in the brain (Hayashi and Takeichi, 2015). PCDH7 is no exception, it was previously named as $\mathrm{BH}-\mathrm{PCDH}$ for brain and heart protocadherin owing to its 
high expression in these tissues (Yoshida et al., 1999). After observing the role of PCDH7 in cultured cells, we aimed to uncover the role of PCDH7 in cell migration in vivo. To this end, we studied the effect of PCDH7 expression upon the neuronal migration in the cerebral cortex using in utero electroporation method. We used in utero electroporation and able to overexpress PCDH7b in ventricular neuronal progenitor cells of E14.5 (Figure 5A). We also expressed GFP marker (pCAGGS_IRES_GFP) to observe the migration of the transfected neurons at 3 days post electroporation (E17.5). In the control (EGFPN1) samples, we observed expected migration of neurons, $40 \%$ of them were able to reach the cortical plate (CP), $50 \%$ of transfected cells migrated along subventricular zone-intermediate zone (SVZ-IZ) and $10 \%$ of them were located in the ventricular zone (VZ) (Figure 5B). Upon overexpression of PCDH7b in mouse embryonic cortex, we observed a significant increase in the proportion of electroporated cells that migrated to the CP. Cells that expressed PCDH7b had an increased rate of migration, hence PCDH7 GFP-positive neurons exited the $\mathrm{VZ}$ and migrated toward the $\mathrm{CP}$ (Figure 5A, lower panel and Figure 5B). This result indicates that overexpression of $\mathrm{PCDH} 7$ in the subventricular neuronal migratory population enhances the migratory behavior of newly born neurons.

\section{Chemical Inhibition of Phosphatase Activity Recovers PCDH7 Knockout Phenotypes}

Our results suggest that enhanced activity of MYPT1 in the absence of PCDH7 affects the p-MLC2 levels at the migrating cell front. To directly test this, we employed calyculin-A, a potent chemical inhibitor of protein phosphatase activity and hypothesized that the enhanced activity of MYPT1 at the cell front can be controlled by limited inhibition of phosphatase activity in the PCDH7 knockout cells which would recover the phenotypes of reduced p-MLC2 levels and the cell motility. Although the $\mathrm{IC}_{50}$ of calyculin-A for PP1 lies in nanomolar range, RPE1 cells exhibited 
rounding behavior and subsequent detachment of the cells in nanomolar concentrations (data not shown). Instead, we used the calyculin-A at picomolar concentration where cell morphologies and migration behavior were not hampered. Indeed, we observed that at $15 \mathrm{pM}$ concentration, myosin phosphorylation at the leading edges were significantly enhanced compared to the knockout (Figure 6A-B). We also checked the recovery of motility phenotype in knockout and observed that at $15 \mathrm{pM}$ calyculin-A, the cell motility is significantly recovered (Video 6 and Figure 6C). Thus, the chemical inhibition of phosphatase activity aids in the recovery of p-Myosin and motility phenotypes which further strengthens the link between PCDH7/Myosin-Phosphatase/Myosin axis and cell motility.

\section{Discussion}

Despite its importance, how the cortex is organized and communicates with the plasma membrane during cell migration is not fully understood. Crosslinked actin network, myosin motors and the associated proteins make up the cortex which lies right under the plasma membrane. The plasticity of the cortex allows the reversible association of the cortex with the plasma membrane and helps in the mechanical transduction of motile cells (Salbreux et al., 2012; Svitkina, 2018). This is indispensable for every step of cell migration: breaking of cell symmetry, protrusion of the leading edge, adhesion to the substrate, contraction and detachment of the rear edge (Petrie et al., 2009). High turn-over of proteins and dynamic interactions in the cortex are thought to contribute to the cortical plasticity; however, its molecular details remain to be determined.

In the present work, we showed that a member of the cadherin superfamily, namely protocadherin7 (PCDH7), displays polarized behavior in a migrating cell with accumulation at the cell leading 
and rear edges. The polarized localization of PCDH7 is both myosin and actin dependent. In a directed migration, cell persistence is defined as the ability of straight-line motion which is correlated with the migration speed through maintenance of cell polarity by actin flow (Maiuri et al., 2015). We showed that the perturbation of PCDH7 through shRNA mediated downregulation or CRISPR/Cas9 based knockout reduces the persistence of cell migration. We further investigated that PCDH7's perturbation reduces phosphorylation of MLC2 and its overexpression enhances phosphorylation of MLC2 at the leading edge. We could not quantify rear edges because of the polarization irregularity of the rear edge, but we think that the similar dynamics of myosin phosphorylation also happens at the trailing rear edge. Myosin motor activity is a highly regulated process that is reversibly controlled by phosphorylation/dephosphorylation of the MLC2. We suggest that PCDH7 tunes Non-Muscle myosin II (NMII) activity at the leading and rear edge to cell polarity and enhances cell persistence. In line with this conclusion, our previous studies demonstrated that PCDH7 functions in building up rounding pressure, which is another myosin II dependent function (Ozlu et al., 2015). In addition, a recent study showed that PCDH7 inhibits homotypic cell-in-cell structure by increasing myosin phosphorylation (Wang et al., 2020).

How does PCDH7 regulate myosin activity during cell migration? To identify the molecular machinery that regulates myosin phosphorylation via PCDH7, we performed a pulldown of PCDH7 followed by LC-MS/MS. As expected, many components of the actomyosin complex were identified. Surprisingly, the complex also precipitated components of phosphatases, including MYPT1, and PP1c $\beta$ which are subunits of myosin phosphatase. MYPT1 is responsible for directing the complex to myosin (MLC2), and PP1c $\beta$ is the catalytic subunit that carries out myosin dephosphorylation (Kiss et al., 2019). We confirmed the interaction between PCDH7 and 
MYPT1 through co-immunoprecipitation, immunofluorescence and Proximity Ligation Assay. We also showed that MYPT1 enrichment on the cell front and the cell rear have a direct correlation with PCDH7 expression; its enrichment is reduced upon knockout and is enhanced upon overexpression. $\mathrm{PP} 1 \alpha$, which is an isoform of $\mathrm{PP} 1 \mathrm{c} \beta$, has previously been shown to interact with PCDH7 through the canonical PP1 $\alpha$ interaction motif (RRVTF) in its cytoplasmic domain (CM3) (Wang et al., 2020; Yoshida et al., 1999). PP1c $\beta$, an isoform of PP1 $\alpha$, also interacts with its regulatory subunits through the RVxF motif (Egloff et al., 1997). The myosin phosphatase targeting subunit, MYPT1 also directly binds to PP1c $\beta$ by its RVxF motif (Kiss et al., 2019). Interestingly, in addition to the longer isoform $(\mathrm{PCDH7c})$, the short version $\mathrm{PCDH7b}$, which does not harbor (RRVTF) motif, also interacted with myosin phosphatase. Therefore, we propose that $\mathrm{PCDH} 7$ (both $\mathrm{b}$ and $\mathrm{c}$ isoforms) and myosin phosphatase interaction is mainly through their direct interactions with MYPT1 subunit in addition to the PP1c $\beta-P C D H 7 c$ interaction. Our immunoprecipitation, PLA and immunofluorescence imaging experiments support interaction between PCDH7 and MYPT1 where both isoforms of PCDH7 recruit MYPT1 to the cell front. However, we cannot exclude the possibility that PCDH7b interacts with myosin phosphatase subunits through cis-interaction with PCDH7c which binds directly to PP1c $\beta$ through its $\mathrm{RVxF}$ motif.

In addition to myosin, our study suggested that another essential component of cell migration, the ERM complex (Yu et al., 2004) is also regulated by PCDH7. Like MLC2, ERM is also a substrate of Myosin Phosphatase (Kiss et al., 2019). Our analysis demonstrated that PCDH7 interacts with ERM and facilitate the recruitment of phosphorylated (activated) Ezrin to the leading edges. The expression of PCDH7 correlated with the enhanced phosphorylation level of ERM at the migratory 
cortex. We propose that MYPT1-PCDH7 interaction contributes to the localized activation of ERM at the migratory cortex. Thus, through the PCDH7-MYPT1-ERM axis PCDH7 acts as a component of the connecting link between the plasma membrane and the actin cytoskeleton which contributes to the establishment of the polarized cortex during migration. We also performed chemical inhibition of myosin phosphatase using Calyculin A as an alternative recovery mode of phenotype. Calyculin treatment on PCDH7 knockout supports that in the absence of PCDH7, it is the enhanced myosin phosphatase activity at the cortex that causes the reduced phosphorylation of MLC2 and leads to impaired migration.

We propose a model for the role of PCDH7 in cell migration and invasion: PCDH7 quenches the activity of myosin phosphatase by interaction with MYPT1. Such a quenching effect on myosin phosphatase will locally limit dephosphorylation of its substrates, myosin and ERM. This enhanced myosin phosphatase activity has the potential to disrupt the existing equilibrium that maintains MLC2 and ERM phosphorylation at the leading edge and the rear, resulting in loss of persistence. An overexpression of $\mathrm{PCDH} 7$ on the other hand, as has been shown in metastatic cancer cell lines previously (Bos et al., 2009; Shishodia et al., 2019) would quench myosin phosphatase activity even further, resulting in enriched phosphorylation of myosin, and thus enhancing the persistence and migration capability.

Recent work showed that PCDH7 determines the morphology of the dendritic spine (Wang et al., 2020). It is plausible that PCDH7 shape the dendritic morphologies or that its orthologues function in retinal axon growth and guidance (Heggem and Bradley, 2003; Leung et al., 2015; Leung et al., 2013; Piper et al., 2008) via a similar mechanism, namely by regulating phosphorylation dynamics 
of MLC2 (Hodges et al., 2011; Rex et al., 2010; Zhang et al., 2005) and ERM. The roles of cadherins in collective cell migration have been increasingly investigated(Theveneau and Mayor, 2012) . OL-pc/PCDH10, a member of the $\delta-2$ protocadherin group has been shown to mediate cell migration when cells are in contact with other cells but not singly (Nakao et al., 2008). Like other members of the cadherin family, PCDH7 is also enriched at cell-cell junctions. As Myosin II A and Myosin IIB isoforms are involved in adherent junctions integrity and biogenesis (Heuze et al., 2019), PCDH7's regulation of myosin activity at cell-cell junctions might contribute to the collective cell migration. More work is required to decipher the role of PCDH7 in collective cell migration. Yet, our study supports the emerging role of protocadherins in migration dependent physiological and pathological processes.

To conclude, we have shown that $\mathrm{PCDH7}$ is an important regulator of directed migration where it displays a polarized localization at the leading and rear edge and spatially regulates myosin and ERM activity. We propose that PCDH7 imparts this regulation by quenching myosin phosphatase at the cell cortex. The model we propose here provides a new component of cell migration that plays an important role in the plasticity of the cell cortex during migration. Further investigation of the molecular mechanisms behind the interaction of PCDH7, myosin phosphatase and myosin will provide important clues about the polarization and the plasticity of the cell migration machinery. It will also shed new light on the signaling pathways behind metastasis.

\section{Materials and Methods}

\section{Plasmids}


PCDH7b and PCDH7cwere cloned into eGFP-N1 vector. For generation of lentiviral vectors, PCDH7b and PCDH7c were cloned into pLenti CMV GFP Puro (addgene\#17448). For PCDH7V5 constructs, PCDH7b and PCDH7c were cloned into PLEX_307 (Addgene\#41392). eGFPPP1CB-MYPT1 and eGFP-PP1CB(D63A)-MYPT1 were provided by Mathieu Bollen from KU, Leuven and generated as previously described (Szklarczyk et al., 2019). Lentiviral constructs expressing Lifeact-RFP, Ezrin-RFP, and Cortactin-RFP were provided by Michael Way from Francis Crick Institute, UK.

\section{Stable cell line generation}

For stable cell line generation using transient transfection, PCDH7 eGFPN1 were transiently transfected in RPE1 cells and selected using G418 supplemented media. Surviving cells were sorted using FACS sorter (SONY SH8000S, benchtop sorter) for GFP expression. Lentiviral packaged particles were used for RPE1 cells and high dose of puromycin $(7 \mu \mathrm{g} / \mathrm{ml})$ was used for overnight selection. Surviving cells showed GFP expression indicating successful transduction and enrichment for PCDH7.

\section{CRISPR/Cas9 mediated knockouts of PCDH7}

Six different guide sequences targeting $\mathrm{PCDH} 7$ were selected based on the recommendation of mit.crispr.edusoftware. Targeting guides with sequences (1) 5'CGACGTCCGCATCGGCAACG-3'， (2) 5'-TTGCCGATGCGGACGTCGGC-3'， (3) 5'CACGTTGCCGATGCGGACGT-3', (4) 5'-GTTGCCGATGCGGACGTCGG-3', , (5) 5'CATCGTGACCGGATCGGGTG-3' (6) 5'-GGCCCCGCCGACGTCCGCAT-3' and nontargeting guide with sequence 5'-ACGGAGGCTAAGCGTCGCAA - 3' were cloned into pLentiCRISPRv2 using BsmBI restriction digestion of the backbone using recommended protocols from Zhang lab (Sanjana et al., 2014). HeLa S3 and RPE1 cells were transiently transfected with 
plentiCRISPRv2 plasmid containing PCDH7 guides and non-targeting control (5'ACGGAGGCTAAGCGTCGCAA - 3'). Monoclonal cells were prepared with dilution and selected for absence of PCDH7 expression using immunoblotting.

\section{shRNA mediated knockdown of PCDH7}

Three separate shRNA vectors targeting PCDH7 were purchased from Thermo Fisher Scientific's GIPZ shRNA library: V3LHS_375966, V3LHS_375967, and V3LHS_152964. Non-silencing shRNA vector was provided along with the GIPZ shRNA vectors. shRNA constructs were packaged into lentiviral particles and transduced cells were monitored between 48-72 hours for turbo-GFP expression.

\section{Single-cell tracking for phenotype assessment}

RPE cells stably expressing shRNAs and RPE1 CRISPR knockout sets were used for single-cell tracking to monitor migration phenotype. We used OlympusPro and Leica DMi8 live imaging microscopes for time-lapse widefield and fluorescence image acquisition of cells. 10x air objective was used for FITC channel. Time between two frames was 10 minutes. Cell migration was recorded for several hours. Custom scripts written in MATLAB (R2017b, The MathWorks) were used to analyze time-lapse videos. After background subtraction and segmentation steps, trajectories of cells were determined by computing mean square displacement in consecutive frames and later used to associate them in each frame. After registering $\mathrm{x}$ and y coordinates of cell, they were used to compute both persistence ratio and angular displacement.

\section{xCELLigence Real Time Cell Migration experiment}

Real time cell migration was performed as recommended using xCELLigence RTCA-DP (ACEA Biosciences Inc., CA, USA). Briefly, 10\% FBS containing DMEM was used at the bottom and 1\% 
FBS DMEM was inserted at the top to create downward chemoattractant gradient. 30,000 cells per cell line were plated on the top chamber in $100 \mu \mathrm{l}$ of medium and let set for 1 hour for the cells to attach to the surface of the top plate. RTCA migration study was performed for 48 hours. 16 well cell invasion and migration (CIM) plates were used for this experiment.

\section{GFP pulldown of PCDH7 isoforms for identification of interactors}

RPE1 cells with stable expression of PCDH7 was used for GFP pulldown using Chromotek GFPTrap Agarose following manufacturer's protocol. On-bead digestion was performed, and samples were used for LC-MS/MS analysis (Q Exactive, Thermo Fisher Scientific). Spectral counts of identified proteins were used to calculate the Bayesian false discovery rate (BFDR) and the probability of possible protein-protein interactions by SAINTexpress v.3.6.3 with -L option. Proteins were filtered out according to the SAINTscore $(>0.5)$ and BFDR value $(<0.05)$. Significant protein hits with 1 SAINTscore were loaded into the STRING database v11.0 (Szklarczyk et al., 2019) by Cytoscape StringApp (Doncheva et al., 2019) with 0.7 confidence. MCL clustering (inflation value: 2.5) of the network was performed by Cytoscape (version 3.7.2) and its plugin clustermaker (Cline et al., 2007) GO and KEGG enrichment analysis of the network was performed via g:Profiler (Raudvere et al., 2019).

\section{Proximity Ligation Assay}

Association of PCDH7 and MYPT1 was examined using the Duolink Proximity Ligation Assay Kit (92101, Sigma-Aldrich) according to the manufacturer's instructions. RPE1 cells expressing PCDH7 were plated on crossbow shaped micropattern coverslips following manufacturer's instructions (10-950-10-18, CYTOO). Two control samples with only primary antibodies (anti- 
MYPT1, sc-514261, Santa Cruz; and anti-GFP, 2555S, Cell Signaling Technology), and one target sample with both primary antibodies were used. For quantification of PLA, cells were imaged in Texas Red channel, DAPI for nucleus and FITC for PCDH7 expression. Data analysis was performed in ImageJ. Briefly, images were processed for finding maxima to highlight the particles. Particle analysis gave the number of PLA dots. Datasets were plotted in PRISM GraphPad and statistical analysis was performed using unpaired $t$-test.

\section{Immunofluorescence}

Antibodies anti-GFP goat (Anthony Hyman lab, in-house preparation, 1:10,000), anti-GFP mouse (11814460001, Roche), anti-MYPT1 mouse (sc-514261, Santa Cruz), anti-pERM rabbit (Cell Signaling Technology 3726s), phalloidin iFlour555 (ab176756, abcam), and phalloidin alexa flour 647 (A22287, Thermo Fisher Scientific) and phospho-myosin light chain 2, S19 mouse (3675, Cell Signaling Technology) were used. DAPI was used for nuclear labeling. Mowiol was used for mounting of coverslips.

\section{Immunoblotting}

Anti-PCDH7 mouse (139274, abcam), anti-Tubulin (3873S, Cell Signaling Technology), antiMYPT1 mouse (sc-514261, Santa Cruz), anti-V5 mouse (Invitrogen R96025), anti-Ezrin (Cell Signaling Technology 3145S), anti-GFP antibodies (Cell Signaling Technology 2555S, and Santa Cruz Sc-8334), and anti-phospho-myosin light chain 2, S19 mouse (3675, Cell Signaling Technology) were used. HRP labelled secondary antibodies were used, anti-mouse (7076 Cell Signaling Technology) and anti-rabbit (7074 Cell signaling technology).

\section{GFP Pulldown of PCDH7-GFP for interaction with Ezrin RFP}


Using Lipofectamine 3000, HeLa S3 cells were transfected with the combinations of PCDH7cEGFP and Ezrin RFP, PCDH7b-EGFP and Ezrin RFP, and empty EGFP and Ezrin RFP (EGFP backbone pLenti CMV GFP Puro was purchased from addgene (Addgene 17448) and pLenti RFP Ezrin was a gift from Michael Way, Crick Institute. Cells were harvested using HBSS solution with calcium and magnesium (Diagnovum D408) and lysed in lysis buffer [pH $7.510 \mathrm{mM}$ Tris, $\left.150 \mathrm{mM} \mathrm{NaCl}, 0.5 \% \mathrm{NP} 40,1.26 \mathrm{mM} \mathrm{CaCl}_{2}, 0.5 \mathrm{mM} \mathrm{MgCl}_{2} .6 \mathrm{H}_{2} \mathrm{O}, 0.4 \mathrm{mM} \mathrm{MgSO} 4.7 \mathrm{H}_{2} \mathrm{O}\right]$ that has been supplemented with protease inhibitor (complete ${ }^{\mathrm{TM}}$, Mini, EDTA-free Protease Inhibitor Cocktail REF 18836170001) and phosSTOP phosphatase inhibitor cocktail (Roche 4906845001] at $4^{0} \mathrm{C}$ for 30 minutes before clearing lysate by centrifugation at $4^{0} \mathrm{C}$. BCA assay was performed for protein quantification. GFP nanotrap agarose beads (Chromotek gta) were used to pulldown GFP and GFP fusion proteins as manufacturer's instructions. Before washing the beads three times with dilution buffer $\left[\mathrm{pH} 7.510 \mathrm{mM}\right.$ Tris, $150 \mathrm{mM} \mathrm{NaCl}, 1.26 \mathrm{mM} \mathrm{CaCl}_{2}, 0.5 \mathrm{mM} \mathrm{MgCl} 2.6 \mathrm{H}_{2} \mathrm{O}$, $0.4 \mathrm{mM} \mathrm{MgSO}_{4} .7 \mathrm{H}_{2} \mathrm{O}$, phosSTOP phosphatase inhibitor cocktail (Roche 4906845001) and protease inhibitor (complete ${ }^{\mathrm{TM}}$, Mini, EDTA-free Protease Inhibitor Cocktail REF 18836170001)], the beads were centrifuged and supernatants of the samples were collected as flowthrough. To elute proteins, the beads were boiled with 1x Laemmli Buffer. Samples as a set of the input, flow through and elution were loaded to the gel and proteins were separated by SDS-PAGE. Immnoblotting was performed using anti-Ezrin (Cell Signaling Technology 3145s), anti-GFP (Thermo Scientific PAI-980A), anti-alpha tubulin (Cell Signaling Technology 3873s). Anti-mouse secondary antibody (Cell Signaling Technology 7076s) and anti-rabbit secondary antibody (Cell Signaling Technology 7074s).

\section{GFP Pull-down of PCDH7c-V5 for interaction with Myosin Phosphatase}


Using Lipofectamine 3000, HeLa S3 cells were transfected with pLenti PCDH7c V5 (backbone Addgene 41392) and, in combination of the wild type Myosin Phosphatase (MP) [EGFP-PP1CBMYPT1], Mutant MP [EGFP-PP1CB-(D63A)-MYPT1], PP1CB [EGFP-PP1CB] and MYPT1 [EGFP-MYPT1] (all cloned into EGFP-C1 backbone). The cells were harvested in HBSS solution with calcium and magnesium (Diagnovum D408) and lysed in the buffer [pH 7.5 10mM Tris, 150 $\mathrm{mM} \mathrm{NaCl}, 0.5 \%$ NP40, $\left.1.26 \mathrm{mM} \mathrm{CaCl}_{2}, 0.5 \mathrm{mM} \mathrm{MgCl} 2.6 \mathrm{H}_{2} \mathrm{O}, 0.4 \mathrm{mM} \mathrm{MgSO} 4.7 \mathrm{H}_{2} \mathrm{O}\right]$ supplemented with protease inhibitor (complete ${ }^{\mathrm{TM}}$, Mini, EDTA-free Protease Inhibitor Cocktail REF 18836170001) and phosSTOP phosphatase inhibitor cocktail (Roche 4906845001$)$ ] at $4^{0} \mathrm{C}$ for 30 minutes before clearing lysate by centrifugation at $4^{0} \mathrm{C}$. BCA assay was performed for protein quantification. GFP nanotrap agarose beads (Chromotek gta) were used for pulldown of GFP and GFP fusion proteins as manufacturer's instructions. Before washing the beads three times with dilution buffer $\left[\mathrm{pH} 7.510 \mathrm{mM}\right.$ Tris, $150 \mathrm{mM} \mathrm{NaCl}, 1.26 \mathrm{mM} \mathrm{CaCl}_{2}, 0.5 \mathrm{mM} \mathrm{MgCl} 2.6 \mathrm{H}_{2} \mathrm{O}$, $0.4 \mathrm{mM} \mathrm{MgSO}_{4} .7 \mathrm{H}_{2} \mathrm{O}$, phosSTOP phosphatase inhibitor cocktail (Roche 4906845001) and protease inhibitor (complete ${ }^{\mathrm{TM}}$, Mini, EDTA-free Protease Inhibitor Cocktail REF 18836170001)], the beads were centrifuged, and supernatants of the samples were collected as flowthrough. To elute proteins, the beads were boiled with 1x Laemmli Buffer. Samples as a set of the input, flow through and elution were loaded to the gel and proteins were separated by SDS-PAGE. Immunoblotting was performed using anti-MYPT1(Santa Cruz sc-514261), anti-V5 (Invitrogen R96025), anti-alpha tubulin (Cell Signaling Technology 3873s), anti-mouse secondary antibody (Cell Signaling Technology 7076s) and anti-rabbit secondary antibody (Cell Signaling Technology 7074s).

\section{V5 Pull-down of PCDH7b-V5 for interaction with Myosin Phosphatase}


Using Lipofectamine 3000, HeLa S3 cells were transfected with pLenti PCDH7b V5 (backbone Addgene 41392) in combination of EGFP-PP1CB, EGFP-MYPT1 and pLenti BirA V5. Cells were harvested separately in 1x HBSS solution with calcium and magnesium (Diagnovum D408) and lysed in the buffer [pH $7.510 \mathrm{mM}$ Tris, $150 \mathrm{mM} \mathrm{NaCl}, 0.5 \% \mathrm{NP} 40,1.26 \mathrm{mM} \mathrm{CaCl} 2,0.5 \mathrm{mM}$ $\left.\mathrm{MgCl}_{2} .6 \mathrm{H}_{2} \mathrm{O}, 0.4 \mathrm{mM} \mathrm{MgSO} 4.7 \mathrm{H}_{2} \mathrm{O}\right]$ supplemented with protease inhibitor (complete ${ }^{\mathrm{TM}}$, Mini, EDTA-free Protease Inhibitor Cocktail REF 18836170001) and phosSTOP phosphatase inhibitor cocktail (Roche 4906845001 )] at $4^{0} \mathrm{C}$ for 30 minutes before clearing lysate by centrifugation at $4^{0} \mathrm{C}$. BCA assay was performed for protein quantification. V5 nanotrap agarose beads (Chromotek V5ta) were used to pulldown V5 fusion proteins as manufacturer's instructions. Before washing the beads three times with dilution buffer $\left[\mathrm{pH} 7.510 \mathrm{mM}\right.$ Tris, $150 \mathrm{mM} \mathrm{NaCl}, 1.26 \mathrm{mM} \mathrm{CaCl}_{2}, 0.5$ $\mathrm{mM} \mathrm{MgCl}_{2} .6 \mathrm{H}_{2} \mathrm{O}, 0.4 \mathrm{mM} \mathrm{MgSO}_{4} .7 \mathrm{H}_{2} \mathrm{O}$, phosSTOP phosphatase inhibitor cocktail (Roche 4906845001) and protease inhibitor $\left(\operatorname{complete}^{\mathrm{\tau м}}\right.$, Mini, EDTA-free Protease Inhibitor Cocktail REF 18836170001)], the beads were centrifuged, and supernatants of the samples were collected as flowthrough. To elute proteins, the beads were boiled with 1x Laemmli Buffer. Samples as a set of the input, flow through and elution were loaded to the gel and proteins were separated by SDS-PAGE. Immunoblotting was performed using anti-GFP (Thermo Scientific PAI-980A), antiGFP (Roche 11814460001), anti-V5 (Invitrogen R96025), anti-alpha tubulin (Cell Signaling Technology 3873s), anti-mouse secondary antibody (Cell Signaling Technology 7076s) and antirabbit secondary antibody (Cell Signaling Technology 7074s).

\section{Immunofluorescence and quantification}

RPE1 cells were seeded to each well of 6 wells plate including $15 \mathrm{~mm}$ coverslip for each sample (Non-targeting (NT), knockout (KO), $\mathrm{KO}+\mathrm{PCDH7b}, \mathrm{KO}+\mathrm{PCDH7c}$, wild type (WT), $\mathrm{WT}+\mathrm{PCDH7c}, \mathrm{WT}+\mathrm{PCDH} 7 \mathrm{~b}$ ) and were grown in DMEM/ F12 that was supplemented with 10\% 
FBS, 1\% penicillin-streptomycin mixture, 1\% L-glutamine. After 24 hours, coverslips were washed with prewarmed 1x HBSS solution (Diagnovum D408) three times and cells on coverslips were fixed with $3 \%$ paraformaldehyde at $37^{\circ} \mathrm{C}$ for 8 minutes. Permeabilization of cells was done with $0.1 \%$ Triton-x in $1 \mathrm{x}$ PBS at room temperature for 5 minutes, and blocking was done with 2 $\%$ BSA $0.02 \% \mathrm{NaN}_{3}$ in $1 \mathrm{x}$ PBS for 1 hour.

Primary antibodies goat anti-GFP (from Anthony Hyman Lab), anti-MYPT1(Santa Cruz sc5142611), anti-P-ERM (Cell Signaling Technology 3726S), anti-pMLC 2(Cell Signaling Technology 3675S,) and secondary antibodies anti-mouse IgG Alexa Flour ${ }^{\circledR} 555$ Fab 2 (Cell Signalling 4409S ), anti-goat 488 (Abcam 150129 ), anti-rabbit IgG Alexa Flour ${ }^{\circledR} 555$ Fab 2 (Cell Signaling Technology 4413S). After washing with 1x PBS three times, nuclei of the cells were stained with Hoechst 33258 (Sigma 861405) at room temperature for 5 minutes. Before mounting and sealing coverslips, they were washed with 1x PBS once.

Imaging was performed by $63 \times$ Plan Apo 1.4 NA oil-immersion objective of Leica DMi8 widefield microscope and Leica LasX software in DAPI (blue), FITC1(green) and Y3(red) fluorescence channels for fixed samples. $Z$ stack was acquired in $3 \mu \mathrm{m}$ range as 8 frames. Maximum projection was performed before quantification.

Properly polarized RPE1 cells that can be distinguished with their cell front and cell rear were chosen for quantification. ImageJ software was used for quantification. In this process, images were firstly converted to 8-bit version. Secondly, a region of interest was determined as a 64 pixel $^{2}$ square by being considered diversity of properly polarized RPE1 cells' size. After five different areas that have both high signal and low signal were determined in cell cortex for the cell front, signals in these regions were measured by five 64 pixel $^{2}$ squares and their averages were calculated for the cell front. After that, signals coming from five different extracellular regions were measured 
by these square and their average was calculated. To eliminate background signal, this value was subtracted from previously calculated averages belonging cell front. Thus, real signal values of them were acquired. This process was repeated by recalling same square for each cell in both same and different sample group to obtain consistent and comparable results.

\section{In-utero electroporation}

In utero electroporation experiment was performed as previously described (Guzelsoy et al., 2019; Saito, 2006). In utero electroporation protocol was carried out using E14.5 pregnant CD1 mice. Briefly, pregnant mice were anesthetized with isoflurane and laparotomy was performed to expose the uterine horns. $500 \mathrm{ng}$ of control (EGFPN1) or PCDH7b-EGFPN1 plasmids was mixed with 500 ng pCAGGS_IRES_GFP marker to observe the transfected cells and $0.1 \%$ Fast Green to monitor the injection (QIAGEN Endofree Plasmid Maxiprep kit were used to prepare all plasmids). Plasmid mixes were injected into lateral ventricle visible through the translucent uterine wall with pulled glass capillaries. After injection, plasmid DNA mix were transfected into the neuronal progenitor cells located in the VZ by electroporation with the settings 0.5 pulses, $30 \mathrm{~V}$, $400 \mathrm{~ms}$ intervals using an electroporater (BTX Harvard Apparatus, ECM830) and $5 \mathrm{~mm}$ tweezertrodes (BTX Harvard Apparatus). Embryos were placed back into the body cavity and allowed to grow for three days. At E17.5, electroporated embryonic brains were collected and fixed with $4 \%$ paraformaldehyde overnight at $4{ }^{\circ} \mathrm{C} .50 \mu \mathrm{m}$ coronal sections of the embryonic brains were prepared using cryostat (CM1950, Leica Biosystems). Sections were stained with anti-GFP (Aves Labs, Cat. \#GFP-1020, 1:1000) antibody to look for the pattern of the migration. Nikon Eclipse 90i confocal microscope was used to capture optical z-series through $50 \mu \mathrm{m}$ images and NIS-Elements AR software was used to generate maximum intensity projections. 10 bins grid was placed onto the images using Adobe Photoshop CC (Adobe Systems) and number of GFP-positive 
cells were counted for each bin with ImageJ (NIH) cell counter option. Sections were stained with anti-GFP (Aves Labs, Cat. \#GFP-1020, 1:1000) antibody to look for the pattern of the migration. Nikon Eclipse 90i confocal microscope was used to capture optical z-series through $50 \mu \mathrm{m}$ images and NIS-Elements AR software was used to generate maximum intensity projections. 10 bins grid was placed onto the images using Adobe Photoshop CC (Adobe Systems) and number of GFPpositive cells were counted for each bin with ImageJ (NIH) cell counter option (85). Data were transferred to Excel (Microsoft Office) for analysis. Bins 1-5 indicate CP, bins 6-9 indicate SVZIZ, and bin 10 indicates VZ. Graphs were plotted using GraphPad Prism 5 software. In utero electroporation protocol was performed in accordance with the guidelines for the care and use of laboratory animals of Koç University and the Ministries of Food, Agriculture and Livestock, Forestry and Water Management of Turkey. CD1 mice were accommodated, sustained, and bred at the Koç University Animal Facility. Ethics approval was received from Koç University Institutional Animal Care and Use Committee.

\section{Live imaging for fluorescent protein localization and perturbation}

RPE1 cells expressing PCDH7c-GFP and lifeact-RFP were plated on ibidi- $\mu$-slide coated with collagen (OLAF pharmaceuticals, GMP Type I Human Collagen). Time lapse imaging was performed using Leica DMI SP8 confocal microscope at $37{ }^{\circ} \mathrm{C}$ in humified chamber. Argon laser and DPSS 561 laser were used for sample excitation and PMT as well as hybrid detectors were used for signal detection. Las X software was used for data collection, and Las X and ImageJ software were used data processing. For perturbation with blebbistatin, medium containing $5 \mu \mathrm{M}$ blebbistatin was added to the imaging chamber.

\section{Statistical Analysis}


For the analysis of single cell tracking experiment, proximity ligation assay, fluorescence quantification and in-utero electroporation, unpaired t-test was used. p-value -0.05 was used as cut-off for significance. Plots were generated using Graphpad PRISM software.

\section{Acknowledgement}

We thank Koc University Proteomics Facility (KUPAM) for proteomics services and support. We thank imaging facility at Koc University (CMIC) for facilitating imaging experiments. We thank Dr. Mathieu Bollen from KU Leuven for supplying MYPT1 and PP1CB fusion vectors. We thank Dr. Michael Way from the Francis Crick Institute for providing lifeact-RFP, and Ezrin-RFP constructs. We thank Aydanur Senturk for her inputs and critical reading of the manuscript. This work is funded by TUBITAK 1001, $116 Z 305$ to NO. H.B. was supported by ITU-BAP grant 202042579.

\section{Author Contribution}

NO and MHQ conceived the project and designed the experiments. MHQ and MTC performed the experiments. EB assisted in the experiments. Analysis of single cell tracking for migration phenotype was performed by HB. Proteomics data analysis was performed by AK. RTCA experiment was performed by ZCUK, in utero experiment was performed by CA. MHQ and NO prepared the manuscript.

\section{Conflict of interest}

The authors declare no conflict of interest.

\section{FIGURE LEGENDS}


Figure 1. PCDH7 localizes at the protruding front and trailing rear edge of migratory RPE1

cells. (A) Representative images from live imaging of an RPE1 cell expressing PCDH7-GFP (green) and lifeact-RFP (red) (Video 1). Upper panel shows an unpolarized RPE1 cell expressing PCDH7 GFP (green) and lifeact RFP (red). As the cell polarized, middle and lower panels show prominent $\mathrm{PCDH} 7$ enrichment on the cell rear and on retraction fibers (magenta arrow) and protruding front edge (white arrow). Scale bar: $20 \mu \mathrm{m}$. (B) Immunostaining of RPE1 cells expressing PCDH7-GFP (green), phosho-Myosin Light Chain 2 (p-MLC2, S19) (red). Scale bars: $20 \mu \mathrm{m}$.

Figure 2. Perturbation of PCDH7 expression affects cell migration persistence and directionality. (A) Immunoblot analysis of PCDH7 from CRISPR based PCDH7 knockout RPE1 cells and the recovered expression of PCDH7 in knockout cells (top). Tubulin is used for loading control (bottom). (B) Representative trajectories from populations of non-targeting (NT), knockout (KO), and add-back of PCDH7 to knockout cells (KO+PCDH7c). (C-G) Measurement of migration parameters upon knockout $(\mathrm{KO})$ and $\mathrm{KO}+\mathrm{PCDH} 7 \mathrm{c}$ for phenotype recovery: (C) Persistence as a ratio of direct distance and total distance (D/T), (D) measures of lower angle displacements $\left(0-36^{\circ}\right)$ and $(\mathbf{E})$ higher angle displacements $\left(144-180^{\circ}\right) \mathrm{n}: \mathrm{NT}=117, \mathrm{KO}=125$, $\mathrm{KO}+\mathrm{PCDH7c}=149 .(\mathrm{F})$ Persistence $(\mathrm{D} / \mathrm{T})$ for $\mathrm{NT}, \mathrm{KO}$, and $\mathrm{KO}+\mathrm{PCDH} 7 \mathrm{~b}$ n: NT=48, KO=41, $\mathrm{KO}+\mathrm{P} 7 \mathrm{~B}=37$. (G) Persistence of RPE cells measured as a ratio of direct distance (D) and total distance $(\mathrm{T})$ covered by individual cells upon shRNA mediating downregulation of PCDH7. $* * * \mathrm{p}<0.001, * * * * \mathrm{p}<0.0001, \quad \mathrm{n}-\operatorname{shRNANS}=94$, shRNA2 $=120$, shRNA3 $=127$. (H) Real Time directed cell migration assay using xCELLigence RTCA for HeLaS3 cells. Blue circles indicate migration of HeLaS3 control cells whereas red squares and green triangles indicate migration 
behavior of two HeLaS3 knockout cells (HeLa KO-1 and HeLa KO-2, derived from two different $\operatorname{sgRNA)~for~} \mathrm{PCDH} 7 * \mathrm{p}<0.05, * * \mathrm{p}<0.01$.

Figure 3. PCDH7 enhances myosin regulatory chain phosphorylation. (A) Immunoblotting analysis of pMLC2(S19) levels in HeLaS3 control (NT) cells, and PCDH7-KO cells. (B) Representative images of the immunofluorescence for pMLC2(S19) in RPE1 cells. NT, KO and recovery cells (KO+PCDH7 isoforms) were used. Cells were stained for pMLC2(S19) (Red), and GFP (green). scale bar $20 \mu \mathrm{m}$. Quantification of the pMLC2(S19) enrichment on cell front (C) in $\mathrm{NT}, \mathrm{KO}$ and recovery $\mathrm{KO}+\mathrm{PCDH} 7$ isoforms. $\mathrm{n}=40$ for each group. $* * * \mathrm{p}<0.001, * * * * \mathrm{p}<0.0001$. (D) Representative immunofluorescence images showing PCDH7-GFP (Green) and p-MLC2 (Red) in wild type cells (top panel) and upon overexpression of PCDH7b-GFP (Middle panel) and PCDH7c-GFP (lower panel). Scale bar $20 \mu \mathrm{m}$ (E) Quantitative comparison of pMLC2 on the cell front in wild type cells and upon overexpression of PCDH7 isoforms, n=40 for each group. $* * * \mathrm{p}<0.001, * * * * \mathrm{p}<0.0001$

Figure 4. PCDH7 interacts with myosin phosphatase subunits. (A) Depiction of relevant clusters of PCDH7 isoform-c interactome determined by GFP mediated affinity pulldown followed by LC-MS/MS. Red inset depicts a set of phosphatase subunits and phosphatase regulatory proteins, green inset highlighting components of myosin complexes, and blue inset showing members of ERM proteins. (B) Immunostaining of RPE1 cells expressing PCDH7-GFP (green), MYPT1 (PPP1R12A) (red) and fluorescent phalloidin (blue). (C) Immunoblotting for GFP pulldown of EGFP-PP1CB-MYPT1 fusion, EGFP-PP1CB*-MYPT1 (D63A) mutant and control construct with cotransfection of PCDH7c-V5 construct. Membranes were blotted using anti-V5, 
anti-MYPT1, anti-GFP and anti-tubulin antibodies. I (Input), FT(Flow Through), E (Elution) (D) Quantification of proximity ligation assay (PLA) showing fluorescence dots per cell for PCDH7GFP (anti-GFP) and MYPT1 (anti-MYPT1) interaction (n=29) along with single primary antibody controls, MYPT1 $(\mathrm{n}=30)$ and GFP $(\mathrm{n}=30) . * * * * \mathrm{p}<0.0001$.

Figure 5: PCDH7 overexpression enhances radial neuronal migration in mouse developing cerebral cortex. (A) Immunofluorescence images of E17.5 coronal cryosections in utero electroporated with EGFPN1 (control) or PCDH7b-EGFPN1 and pCAGGS_IRES_GFP at E14.5. GFP immunostaining was performed to visualize migration pattern of transfected cells and DAPI was used to stain nucleus. VZ, ventricular zone; SVZ-IZ, subventricular zone-intermediate zone; CP, cortical plate; MZ, marginal zone. Scale bar, $100 \mu \mathrm{m}$. (B) Quantification of in utero electroporation experiment of PCDH7b. Cortical sections were divided into zones and normalized percentages of GFP-positive neurons counted in each zone are plotted as bar graphs. (For PCDH7b; a total of $n=3373$ for EGFPN1, $n=3316$ from 2 biological replicates were included. Lines represent S.E.M. Unpaired two-tailed $t$ test, $\mathrm{p}$ value: ${ }^{*} \mathrm{p}<0.05,{ }^{*} \mathrm{p}<0.01$.

Figure 6: Chemical inhibition of phosphatase activity recovers reduced pMLC2 levels and persistence of migration in KO cells. (A) Immunofluorescence images showing pMLC2 (S19) on cell front in different groups; NT, KO, and KO + Calyculin A (15 pM). (B) Quantitative comparison of pMLC2(S19) on cell front in different groups NT, KO, and $\mathrm{KO}+$ Calyculin A, $\mathrm{n}=40$ for each group. Scale bar $20 \mu \mathrm{m}$. (C) Comparison of persistence of migration in different 
groups: $\mathrm{NT}, \mathrm{KO}$, and $\mathrm{KO}+$ Calyculin $\mathrm{A}(15 \mathrm{pM}) . \mathrm{n}: \mathrm{NT}=63, \mathrm{KO}=63$, Calyculin $\mathrm{A}=69$. p value: $* \mathrm{p}<0.05, * * * \mathrm{p}<0.001, * * * * \mathrm{p}<0.0001$

Figure 7. A model describing new roles of PCDH7 during cell migration

Left panel shows wild type PCDH7 expressing cell. PCDH7 enriches on the leading edge. Through its quenching effect on myosin phosphatase, it creates a zone of reduced phosphatase activity resulting in higher MLC2 phosphorylation. Equilibrium favors a maintained phosphorylated MLC2 and phosphorylated ERM at the leading and rear edges which contributes to actomyosin contractile ability that drives persistent migration. Right panel depicts PCDH7 knockout scenario. Upon removal of $\mathrm{PCDH} 7$, the quenching of myosin phosphatase is no longer present. Free and active myosin phosphatase disrupts equilibrium to lower levels of MLC2 and ERM phosphorylation. Affected protrusion and retraction results in reduced persistence.

Figure S1. PCDH7 and F-actin colocalize in unpolarized and polarized cells. (A) Representative images of video 2, where a PCDH7-GFP and lifeact-RFP expressing cell shows co-localization of PCDH7 and F-actin at the cell leading (white) and rear (magenta) edges. Representative images from live imaging of PCDH7-GFP and lifeact-RFP expressing RPE1 cells before (top) and 45 minutes after (bottom) blebbistatin treatment. Scale bars, $20 \mu \mathrm{m}$.

Figure S2. shRNA mediated perturbation of PCDH7 affects cell migration persistence and directionality (A) PCDH7 expression was perturbed using shRNA in RPE cells. Immunoblot showing PCDH7 expression in control (non-treated), shRNA NS (treated with non-silencing shRNA) and shRNA1, 2 and 3 against PCDH7 (top). Tubulin antibody is used for loading control 
(bottom). (B) Representative trajectories from shRNA NS (control) and shRNA2 (PCDH7) and shRNA3 (PCDH7) treated cell populations. Measures of angular displacement phenotype upon shRNA treatment (C-D): (C) Lower angle displacements $\left(0-36^{\circ}\right)$ and (D) higher angle displacements $\left(144-180^{\circ}\right) . * * * \mathrm{p}<0.001, * * * * \mathrm{p}<0.0001, \quad \mathrm{n}:$ shRNANS $=94$, shRNA2=120, shRNA3=127. (E) Generation of PCDH7 knockout in HeLaS3 cells. Two different guide RNAs were used for the generation of two different clones. KO-1 and $\mathrm{KO}-2$ were confirmed using PCDH7 immunoblotting taking tubulin as loading control.

Figure S3. PCDH7 expression correlates with the enrichment of the MYPT1 component of myosin phosphatase.

(A) Immunofluorescence results showing representative images of MYPT1 and PCDH7-GFP in non-targeting (NT), knockout (KO), and upon recovery of isoforms (PCDH7c, PCDH7b) in the knockout. (B) Quantification of MYPT1 enrichment on the cell front and in different groups: nontargeting, knockout, and isoforms (PCDH7b and PCDH7c). (C) Representative immunofluorescence images showing PCDH7-GFP (Green) and MYPT1 (Red) in wild type cells (top panel) and upon overexpression of PCDH7b-GFP (Middle panel) and PCDH7c-GFP (lower panel). (D) Quantitative comparison of MYPT1 in wild type cells and upon overexpression of PCDH7 isoforms on the cell front. For quantification, $\mathrm{n}=40$ for each group. $* * \mathrm{p}<0.01$, $* * * \mathrm{p}<0.001, * * * * \mathrm{p}<0.0001$. Scale bar $20 \mu \mathrm{m}$

Figure S4. PCDH7 isoforms interact with components of Myosin Phosphatase.

(A) Immunoblotting for GFP pulldown of EGFP-MYPT1 fusion, EGFP-PP1CB and control construct with cotransfection of PCDH7c-V5. Membranes were blotted using anti-V5 (for 
PCDH7c-V5), anti-MYPT1, anti-GFP (for EGFP-PP1CB, and empty GFP), and anti-Tubulin antibodies. (B) Representative images from proximity ligation assay depicting fluorescent signal from interaction of MYPT1 and PCDH7-GFP (Red dots, upper panel) as well as the control groups (middle and lower panels). Scale bar $20 \mu \mathrm{m}$. (C) immunoblotting for V5 pulldown PCDH7-b V5, cotransfected with EGFP-MYPT1 and control construct BirA-V5. Membranes were blotted using anti-V5 (for PCDH7b-V5 and BirA-V5), anti-GFP (for EGFP-MYPT1), and anti-Tubulin antibodies. (D) Immunoblotting for V5 pulldown PCDH7-b V5, cotransfected with EGFP-PP1CB and control construct BirA-V5. Membranes were blotted using anti-V5 (for PCDH7b-V5 and BirA-V5), anti-GFP (EGFP-PP1CB), and anti-Tubulin antibodies.

Figure S5: PCDH7 interacts with Ezrin and PCDH7 expression correlates with ERM phosphorylation on the cell front. (A) Immunofluorescence of RPE1 cell expressing PCDH7bGFP (Green) and phospho-ERM (Red), scale bar $20 \mu \mathrm{m}$. (B) Immunoblotting of GFP pulldown of PCDH7c-GFP and Ezrin-RFP expressing cells using antibodies against PCDH7, Ezrin, Tubulin and GFP. (C) Representative immunofluorescence images of NT, KO and upon PCDH7 isoforms recovery showing PCDH7-GFP (Green), p-ERM (Red), phalloidin (Magenta). Scale bar $20 \mu \mathrm{m}$. (D) Quantification of p-ERM enrichment on the cell front in different groups: NT, KO, and recovery in $\mathrm{KO}$ of isoforms $\mathrm{PCDH7c}$ and $\mathrm{PCDH7b} \mathrm{n}=40$ for each group. $* * \mathrm{p}<0.01$, $* * * * \mathrm{p}<0.0001$

Video 1: Confocal scanning imaging of RPE1 cell expressing PCDH7c-GFP and lifeact-RFP. Individual frames were collected 10 minutes apart. 
Video 2: Confocal scanning imaging of RPE1 cell expressing PCDH7c-GFP and lifeact-RFP.

Individual frames were collected $\sim 2$ seconds apart.

Video 3: Blebbistatin treatment of RPE1 cell expressing PCDH7c-GFP and lifeact-RFP.

Video 4: Single cell tracking of RPE1 cells upon PCDH7 knockout and recovery.

Video 5: RPE1 cells expressing PCDH7c-GFP and Ezrin-RFP.

Video 6: Single Cell tracking of RPE1 cells upon PCDH7 knockout and recovery upon calyculinA mediated inhibition of myosin phosphatase.

Supplementary Table 1. Proteins identified as interactors upon PCDH7c-GFP pulldown followed by LC-MS/MS.

\section{References}

Arpin, M., D. Chirivino, A. Naba, and I. Zwaenepoel. 2011. Emerging role for ERM proteins in cell adhesion and migration. Cell Adh Migr. 5:199-206.

Baumgartner, M., A.L. Sillman, E.M. Blackwood, J. Srivastava, N. Madson, J.W. Schilling, J.H. Wright, and D.L. Barber. 2006. The Nck-interacting kinase phosphorylates ERM proteins for formation of lamellipodium by growth factors. Proc Natl Acad Sci U S A. 103:13391-13396.

Bos, P.D., X.H. Zhang, C. Nadal, W. Shu, R.R. Gomis, D.X. Nguyen, A.J. Minn, M.J. van de Vijver, W.L. Gerald, J.A. Foekens, and J. Massague. 2009. Genes that mediate breast cancer metastasis to the brain. Nature. 459:1005-1009.

Bosk, S., J.A. Braunger, V. Gerke, and C. Steinem. 2011. Activation of F-actin binding capacity of ezrin: synergism of PIP(2) interaction and phosphorylation. Biophys J. 100:1708-1717.

Budnar, S., and A.S. Yap. 2013. A mechanobiological perspective on cadherins and the actin-myosin cytoskeleton. F1000Prime Rep. 5:35.

Cai, Y., N. Biais, G. Giannone, M. Tanase, G. Jiang, J.M. Hofman, C.H. Wiggins, P. Silberzan, A. Buguin, B. Ladoux, and M.P. Sheetz. 2006. Nonmuscle myosin IIA-dependent force inhibits cell spreading and drives F-actin flow. Biophys J. 91:3907-3920.

Chen, Q., A. Boire, X. Jin, M. Valiente, E.E. Er, A. Lopez-Soto, L. Jacob, R. Patwa, H. Shah, K. Xu, J.R. Cross, and J. Massague. 2016. Carcinoma-astrocyte gap junctions promote brain metastasis by cGAMP transfer. Nature. 533:493-498.

Cline, M.S., M. Smoot, E. Cerami, A. Kuchinsky, N. Landys, C. Workman, R. Christmas, I. Avila-Campilo, M. Creech, B. Gross, K. Hanspers, R. Isserlin, R. Kelley, S. Killcoyne, S. Lotia, S. Maere, J. Morris, K. Ono, V. Pavlovic, A.R. Pico, A. Vailaya, P.L. Wang, A. Adler, B.R. Conklin, L. Hood, M. Kuiper, C. 
Sander, I. Schmulevich, B. Schwikowski, G.J. Warner, T. Ideker, and G.D. Bader. 2007. Integration of biological networks and gene expression data using Cytoscape. Nat Protoc. 2:2366-2382.

Conti, M.A., and R.S. Adelstein. 2008. Nonmuscle myosin II moves in new directions. J Cell Sci. 121:11-18.

Cramer, L.P. 1999. Organization and polarity of actin filament networks in cells: implications for the mechanism of myosin-based cell motility. Biochem Soc Symp. 65:173-205.

Cramer, L.P. 2013. Mechanism of cell rear retraction in migrating cells. Curr Opin Cell Biol. 25:591-599.

Cramer, L.P., M. Siebert, and T.J. Mitchison. 1997. Identification of novel graded polarity actin filament bundles in locomoting heart fibroblasts: implications for the generation of motile force. $J$ Cell Biol. 136:1287-1305.

Doncheva, N.T., J.H. Morris, J. Gorodkin, and L.J. Jensen. 2019. Cytoscape StringApp: Network Analysis and Visualization of Proteomics Data. J Proteome Res. 18:623-632.

Egloff, M.P., D.F. Johnson, G. Moorhead, P.T. Cohen, P. Cohen, and D. Barford. 1997. Structural basis for the recognition of regulatory subunits by the catalytic subunit of protein phosphatase 1. EMBO J. 16:1876-1887.

Guzelsoy, G., C. Akkaya, D. Atak, C.D. Dunn, A. Kabakcioglu, N. Ozlu, and G. Ince-Dunn. 2019. Terminal neuron localization to the upper cortical plate is controlled by the transcription factor NEUROD2. Sci Rep. 9:19697.

Hanahan, D., and R.A. Weinberg. 2011. Hallmarks of cancer: the next generation. Cell. 144:646-674.

Hayashi, S., Y. Inoue, H. Kiyonari, T. Abe, K. Misaki, H. Moriguchi, Y. Tanaka, and M. Takeichi. 2014. Protocadherin-17 mediates collective axon extension by recruiting actin regulator complexes to interaxonal contacts. Dev Cell. 30:673-687.

Hayashi, S., and M. Takeichi. 2015. Emerging roles of protocadherins: from self-avoidance to enhancement of motility. J Cell Sci. 128:1455-1464.

Heggem, M.A., and R.S. Bradley. 2003. The cytoplasmic domain of Xenopus NF-protocadherin interacts with TAF1/set. Dev Cell. 4:419-429.

Heuze, M.L., G.H.N. Sankara Narayana, J. D'Alessandro, V. Cellerin, T. Dang, D.S. Williams, J.C. Van Hest, P. Marcq, R.M. Mege, and B. Ladoux. 2019. Myosin II isoforms play distinct roles in adherens junction biogenesis. Elife. 8.

Hipfner, D.R., N. Keller, and S.M. Cohen. 2004. Slik Sterile-20 kinase regulates Moesin activity to promote epithelial integrity during tissue growth. Genes Dev. 18:2243-2248.

Hodges, J.L., K. Newell-Litwa, H. Asmussen, M. Vicente-Manzanares, and A.R. Horwitz. 2011. Myosin IIb activity and phosphorylation status determines dendritic spine and post-synaptic density morphology. PLoS One. 6:e24149.

Houdusse, A., and H.L. Sweeney. 2016. How Myosin Generates Force on Actin Filaments. Trends Biochem Sci. 41:989-997.

Ikebe, M., D.J. Hartshorne, and M. Elzinga. 1986. Identification, phosphorylation, and dephosphorylation of a second site for myosin light chain kinase on the 20,000-dalton light chain of smooth muscle myosin. J Biol Chem. 261:36-39.

Ito, M., T. Nakano, F. Erdodi, and D.J. Hartshorne. 2004. Myosin phosphatase: structure, regulation and function. Mol Cell Biochem. 259:197-209.

Jeanes, A., C.J. Gottardi, and A.S. Yap. 2008. Cadherins and cancer: how does cadherin dysfunction promote tumor progression? Oncogene. 27:6920-6929.

Jontes, J.D. 2016. The Nonclustered Protocadherins. In The Cadherin Superfamily: Key Regulators of Animal Development and Physiology. S.T. Suzuki and S. Hirano, editors. Springer Japan, Tokyo. 223-249.

Juanes-Garcia, A., J.R. Chapman, R. Aguilar-Cuenca, C. Delgado-Arevalo, J. Hodges, L.A. Whitmore, J. Shabanowitz, D.F. Hunt, A.R. Horwitz, and M. Vicente-Manzanares. 2015. A regulatory motif in nonmuscle myosin II-B regulates its role in migratory front-back polarity. J Cell Biol. 209:23-32. 
Kiss, A., F. Erdodi, and B. Lontay. 2019. Myosin phosphatase: Unexpected functions of a long-known enzyme. Biochim Biophys Acta Mol Cell Res. 1866:2-15.

Leung, L.C., W.A. Harris, C.E. Holt, and M. Piper. 2015. NF-Protocadherin Regulates Retinal Ganglion Cell Axon Behaviour in the Developing Visual System. PLoS One. 10:e0141290.

Leung, L.C., V. Urbancic, M.L. Baudet, A. Dwivedy, T.G. Bayley, A.C. Lee, W.A. Harris, and C.E. Holt. 2013. Coupling of NF-protocadherin signaling to axon guidance by cue-induced translation. Nat Neurosci. 16:166-173.

Maiuri, P., J.F. Rupprecht, S. Wieser, V. Ruprecht, O. Benichou, N. Carpi, M. Coppey, S. De Beco, N. Gov, C.P. Heisenberg, C. Lage Crespo, F. Lautenschlaeger, M. Le Berre, A.M. Lennon-Dumenil, M. Raab, H.R. Thiam, M. Piel, M. Sixt, and R. Voituriez. 2015. Actin flows mediate a universal coupling between cell speed and cell persistence. Cell. 161:374-386.

Mendonsa, A.M., T.Y. Na, and B.M. Gumbiner. 2018. E-cadherin in contact inhibition and cancer. Oncogene. 37:4769-4780.

Moeller, M.J., A. Soofi, G.S. Braun, X. Li, C. Watzl, W. Kriz, and L.B. Holzman. 2004. Protocadherin FAT1 binds Ena/VASP proteins and is necessary for actin dynamics and cell polarization. EMBO J. 23:3769-3779.

Mrozik, K.M., O.W. Blaschuk, C.M. Cheong, A.C.W. Zannettino, and K. Vandyke. 2018. N-cadherin in cancer metastasis, its emerging role in haematological malignancies and potential as a therapeutic target in cancer. BMC Cancer. 18:939.

Nakao, S., A. Platek, S. Hirano, and M. Takeichi. 2008. Contact-dependent promotion of cell migration by the OL-protocadherin-Nap1 interaction. J Cell Biol. 182:395-410.

Ozlu, N., M.H. Qureshi, Y. Toyoda, B.Y. Renard, G. Mollaoglu, N.E. Ozkan, S. Bulbul, I. Poser, W. Timm, A.A. Hyman, T.J. Mitchison, and J.A. Steen. 2015. Quantitative comparison of a human cancer cell surface proteome between interphase and mitosis. EMBO J. 34:251-265.

Petrie, R.J., A.D. Doyle, and K.M. Yamada. 2009. Random versus directionally persistent cell migration. Nat Rev Mol Cell Biol. 10:538-549.

Piper, M., A. Dwivedy, L. Leung, R.S. Bradley, and C.E. Holt. 2008. NF-protocadherin and TAF1 regulate retinal axon initiation and elongation in vivo. $J$ Neurosci. 28:100-105.

Ponti, A., M. Machacek, S.L. Gupton, C.M. Waterman-Storer, and G. Danuser. 2004. Two distinct actin networks drive the protrusion of migrating cells. Science. 305:1782-1786.

Prag, S., M. Parsons, M.D. Keppler, S.M. Ameer-Beg, P. Barber, J. Hunt, A.J. Beavil, R. Calvert, M. Arpin, B. Vojnovic, and T. Ng. 2007. Activated ezrin promotes cell migration through recruitment of the GEF Dbl to lipid rafts and preferential downstream activation of Cdc42. Mol Biol Cell. 18:29352948.

Qiu, C., X. Bu, and Z. Jiang. 2016. Protocadherin-10 acts as a tumor suppressor gene, and is frequently downregulated by promoter methylation in pancreatic cancer cells. Oncol Rep. 36:383-389.

Ramakrishnan, N.A., M.J. Drescher, K.M. Khan, J.S. Hatfield, and D.G. Drescher. 2012. HCN1 and HCN2 proteins are expressed in cochlear hair cells: HCN1 can form a ternary complex with protocadherin 15 CD3 and F-actin-binding filamin A or can interact with HCN2. J Biol Chem. 287:37628-37646.

Ratheesh, A., and A.S. Yap. 2012. A bigger picture: classical cadherins and the dynamic actin cytoskeleton. Nat Rev Mol Cell Biol. 13:673-679.

Raudvere, U., L. Kolberg, I. Kuzmin, T. Arak, P. Adler, H. Peterson, and J. Vilo. 2019. g:Profiler: a web server for functional enrichment analysis and conversions of gene lists (2019 update). Nucleic Acids Res. 47:W191-W198.

Rex, C.S., C.F. Gavin, M.D. Rubio, E.A. Kramar, L.Y. Chen, Y. Jia, R.L. Huganir, N. Muzyczka, C.M. Gall, C.A. Miller, G. Lynch, and G. Rumbaugh. 2010. Myosin Ilb regulates actin dynamics during synaptic plasticity and memory formation. Neuron. 67:603-617. 
Ridley, A.J., M.A. Schwartz, K. Burridge, R.A. Firtel, M.H. Ginsberg, G. Borisy, J.T. Parsons, and A.R. Horwitz. 2003. Cell migration: integrating signals from front to back. Science. 302:1704-1709.

Saito, T. 2006. In vivo electroporation in the embryonic mouse central nervous system. Nat Protoc. 1:1552-1558.

Salbreux, G., G. Charras, and E. Paluch. 2012. Actin cortex mechanics and cellular morphogenesis. Trends Cell Biol. 22:536-545.

Sanjana, N.E., O. Shalem, and F. Zhang. 2014. Improved vectors and genome-wide libraries for CRISPR screening. Nat Methods. 11:783-784.

Schwartz, M.A., and A.R. Horwitz. 2006. Integrating adhesion, protrusion, and contraction during cell migration. Cell. 125:1223-1225.

Shirazi, A., K. lizuka, P. Fadden, C. Mosse, A.P. Somlyo, A.V. Somlyo, and T.A. Haystead. 1994. Purification and characterization of the mammalian myosin light chain phosphatase holoenzyme. The differential effects of the holoenzyme and its subunits on smooth muscle. J Biol Chem. 269:31598-31606.

Shishodia, G., S. Koul, and H.K. Koul. 2019. Protocadherin 7 is overexpressed in castration resistant prostate cancer and promotes aberrant MEK and AKT signaling. Prostate. 79:1739-1751.

Somlyo, A.P., and A.V. Somlyo. 2003. Ca2+ sensitivity of smooth muscle and nonmuscle myosin II: modulated by G proteins, kinases, and myosin phosphatase. Physiol Rev. 83:1325-1358.

Straight, A.F., A. Cheung, J. Limouze, I. Chen, N.J. Westwood, J.R. Sellers, and T.J. Mitchison. 2003. Dissecting temporal and spatial control of cytokinesis with a myosin II Inhibitor. Science. 299:1743-1747.

Svitkina, T. 2018. The Actin Cytoskeleton and Actin-Based Motility. Cold Spring Harb Perspect Biol. 10. Szklarczyk, D., A.L. Gable, D. Lyon, A. Junge, S. Wyder, J. Huerta-Cepas, M. Simonovic, N.T. Doncheva, J.H. Morris, P. Bork, L.J. Jensen, and C.V. Mering. 2019. STRING v11: protein-protein association networks with increased coverage, supporting functional discovery in genome-wide experimental datasets. Nucleic Acids Res. 47:D607-D613.

Te Boekhorst, V., L. Preziosi, and P. Friedl. 2016. Plasticity of Cell Migration In Vivo and In Silico. Annu Rev Cell Dev Biol. 32:491-526.

Theveneau, E., and R. Mayor. 2012. Cadherins in collective cell migration of mesenchymal cells. Curr Opin Cell Biol. 24:677-684.

Uretmen Kagiali, Z.C., N. Saner, M. Akdag, E. Sanal, B.S. Degirmenci, G. Mollaoglu, and N. Ozlu. 2020. CLIC4 and CLIC1 bridge plasma membrane and cortical actin network for a successful cytokinesis. Life Sci Alliance. 3.

Vicente-Manzanares, M., M.A. Koach, L. Whitmore, M.L. Lamers, and A.F. Horwitz. 2008. Segregation and activation of myosin IIB creates a rear in migrating cells. J Cell Biol. 183:543-554.

Vicente-Manzanares, M., X. Ma, R.S. Adelstein, and A.R. Horwitz. 2009. Non-muscle myosin II takes centre stage in cell adhesion and migration. Nat Rev Mol Cell Biol. 10:778-790.

Vicente-Manzanares, M., J. Zareno, L. Whitmore, C.K. Choi, and A.F. Horwitz. 2007. Regulation of protrusion, adhesion dynamics, and polarity by myosins IIA and IIB in migrating cells. $J$ Cell Biol. 176:573-580.

Wang, C., A. Chen, B. Ruan, Z. Niu, Y. Su, H. Qin, Y. Zheng, B. Zhang, L. Gao, Z. Chen, H. Huang, X. Wang, and Q. Sun. 2020. PCDH7 Inhibits the Formation of Homotypic Cell-in-Cell Structure. Front Cell Dev Biol. 8:329.

Yasuda, S., H. Tanaka, H. Sugiura, K. Okamura, T. Sakaguchi, U. Tran, T. Takemiya, A. Mizoguchi, Y. Yagita, T. Sakurai, E.M. De Robertis, and K. Yamagata. 2007. Activity-induced protocadherin arcadlin regulates dendritic spine number by triggering $\mathrm{N}$-cadherin endocytosis via TAO2beta and $\mathrm{p} 38$ MAP kinases. Neuron. 56:456-471. 
Yoshida, K., M. Watanabe, H. Kato, A. Dutta, and S. Sugano. 1999. BH-protocadherin-c, a member of the cadherin superfamily, interacts with protein phosphatase 1 alpha through its intracellular domain. FEBS Lett. 460:93-98.

Yu, Y., J. Khan, C. Khanna, L. Helman, P.S. Meltzer, and G. Merlino. 2004. Expression profiling identifies the cytoskeletal organizer ezrin and the developmental homeoprotein Six-1 as key metastatic regulators. Nat Med. 10:175-181.

Zhang, D., W. Zhao, X. Liao, T. Bi, H. Li, and X. Che. 2012. Frequent silencing of protocadherin 8 by promoter methylation, a candidate tumor suppressor for human gastric cancer. Oncol Rep. 28:1785-1791.

Zhang, H., D.J. Webb, H. Asmussen, S. Niu, and A.F. Horwitz. 2005. A GIT1/PIX/Rac/PAK signaling module regulates spine morphogenesis and synapse formation through MLC. J Neurosci. 25:3379-3388.

Zhou, X., B.L. Updegraff, Y. Guo, M. Peyton, L. Girard, J.E. Larsen, X.J. Xie, Y. Zhou, T.H. Hwang, Y. Xie, J. Rodriguez-Canales, P. Villalobos, C. Behrens, Wistuba, II, J.D. Minna, and K.A. O'Donnell. 2017. PROTOCADHERIN 7 Acts through SET and PP2A to Potentiate MAPK Signaling by EGFR and KRAS during Lung Tumorigenesis. Cancer Res. 77:187-197.

Zhu, P., J. Lv, Z. Yang, L. Guo, L. Zhang, M. Li, W. Han, X. Chen, H. Zhuang, and F. Lu. 2014. Protocadherin 9 inhibits epithelial-mesenchymal transition and cell migration through activating GSK-3beta in hepatocellular carcinoma. Biochem Biophys Res Commun. 452:567-574. 
Qureshi et. al., Figure 1

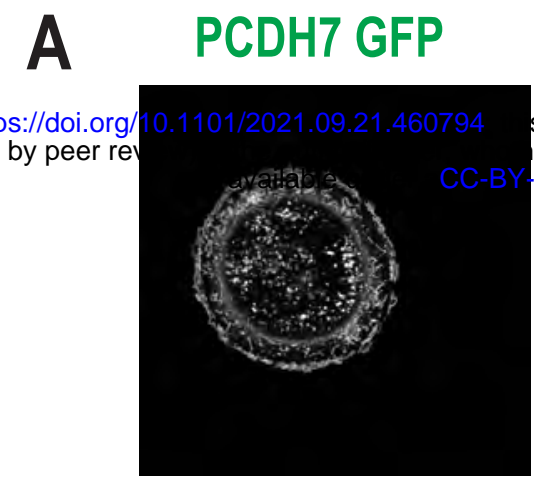

Lifeact-RFP

Merge

bioRxiv preprint doi: https://doi.org/ (which was not certified by peer re
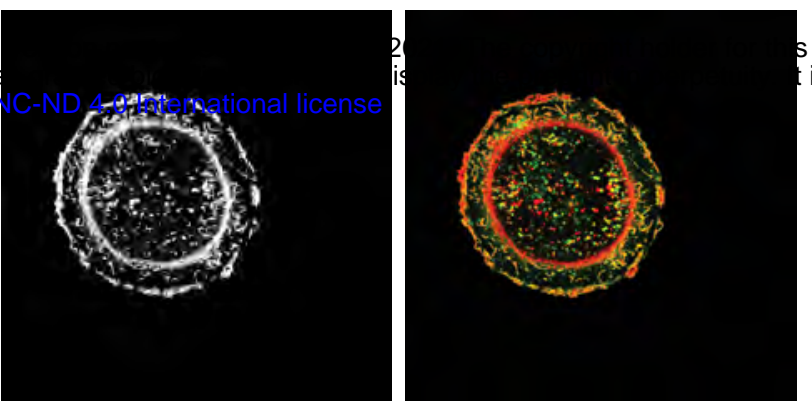

preprint is made
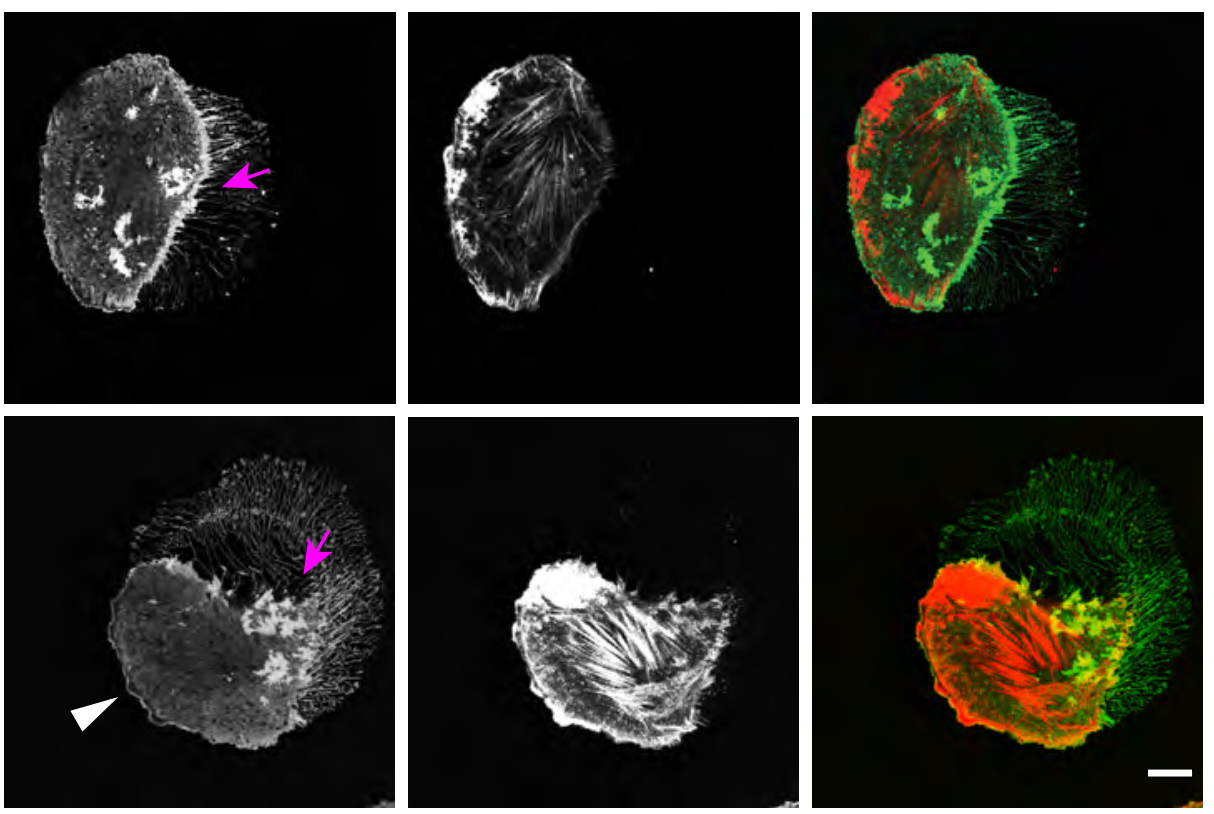

B

PCDH7 GFP

p-MLC2

Merge

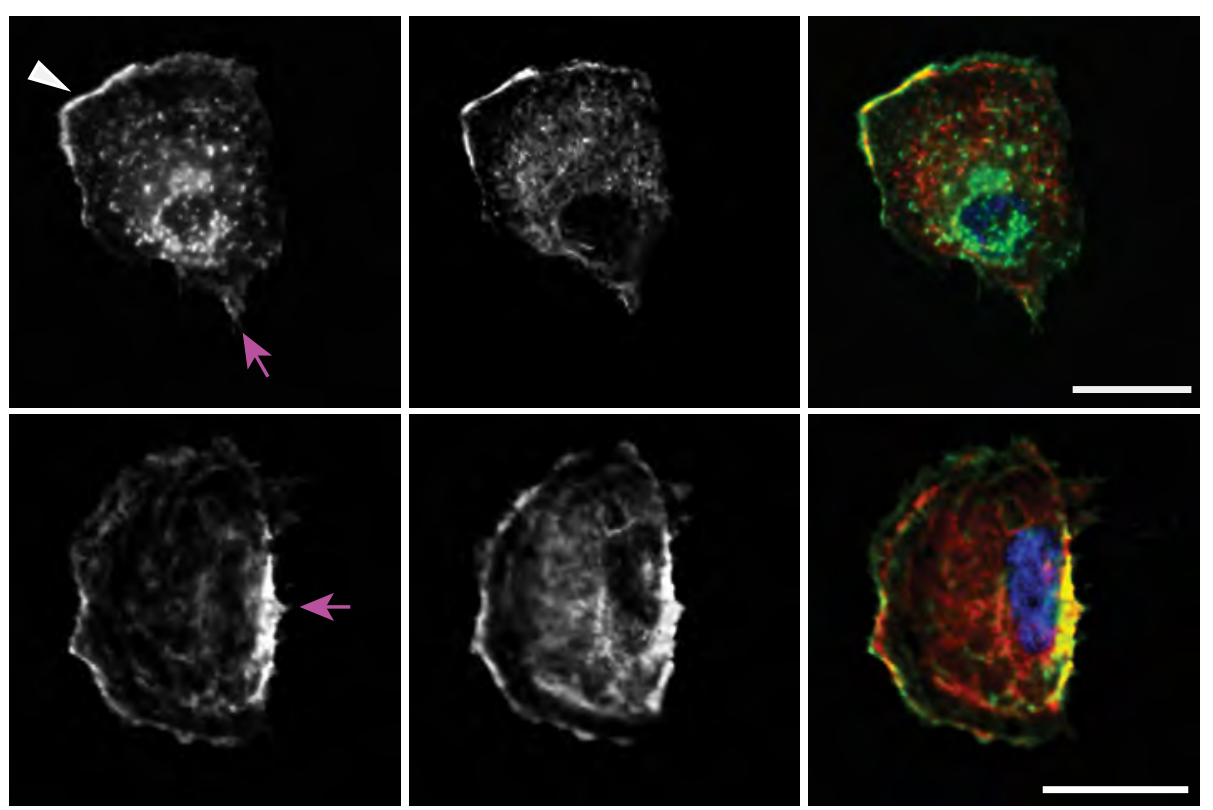


Qureshi et. al., Figure 2

A

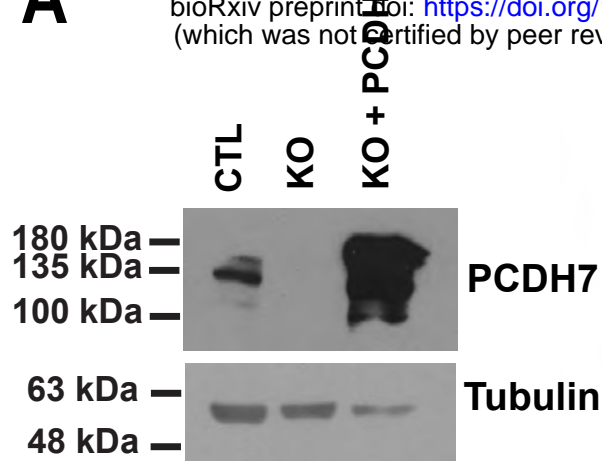

Bio1 101/2021.09.21.460794; this version posted September 21, 2021. The copyright holder for this preprint

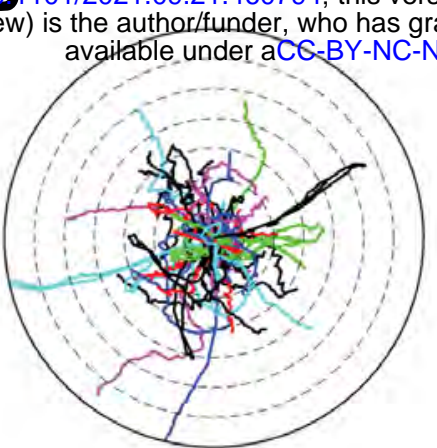

NT

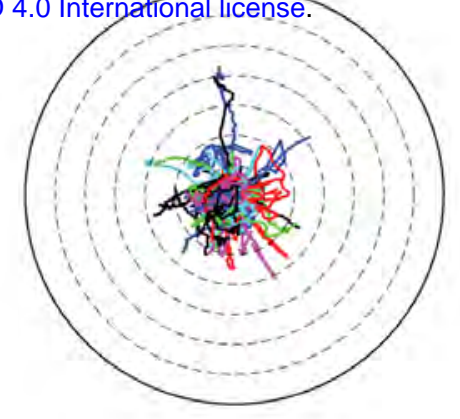

KO

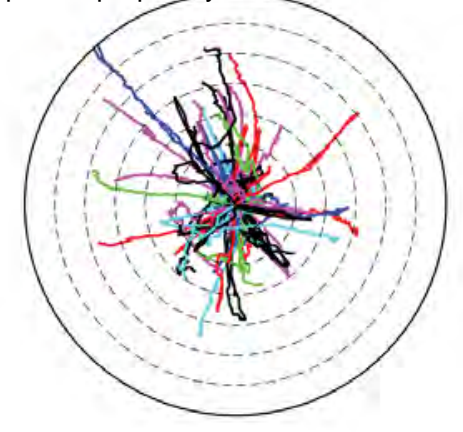

$\mathrm{KO}+\mathrm{PCDH7c}$
C

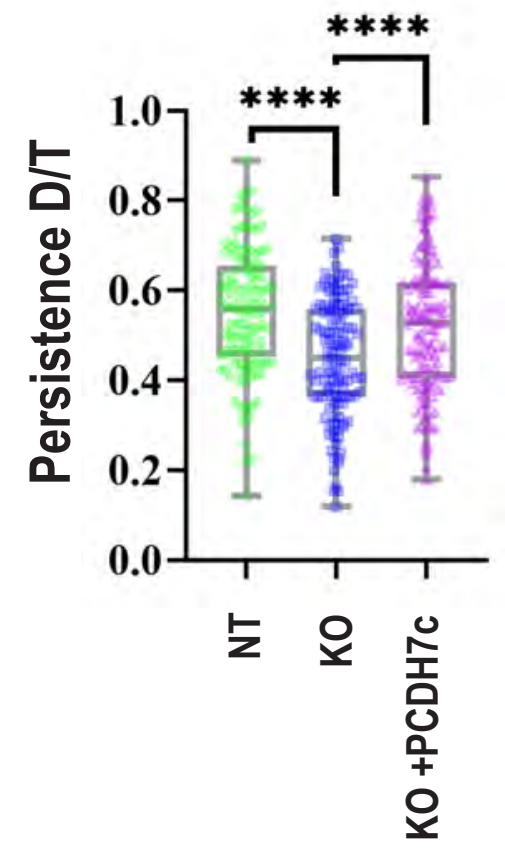

$\mathbf{F}$

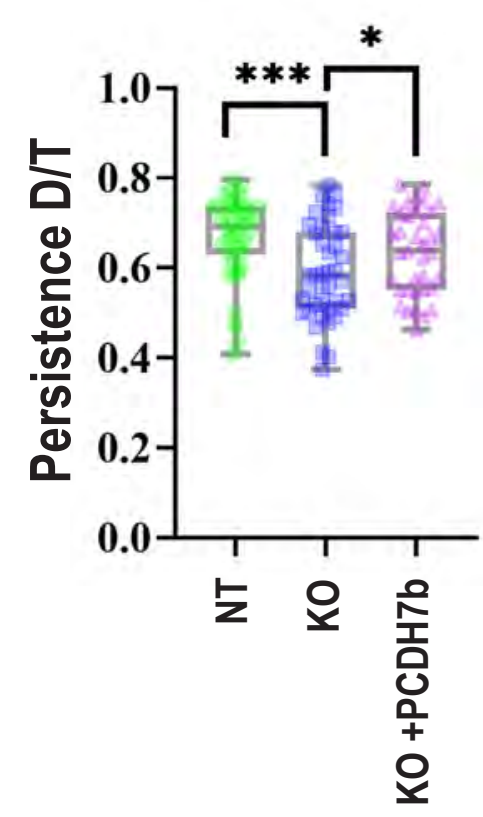

D

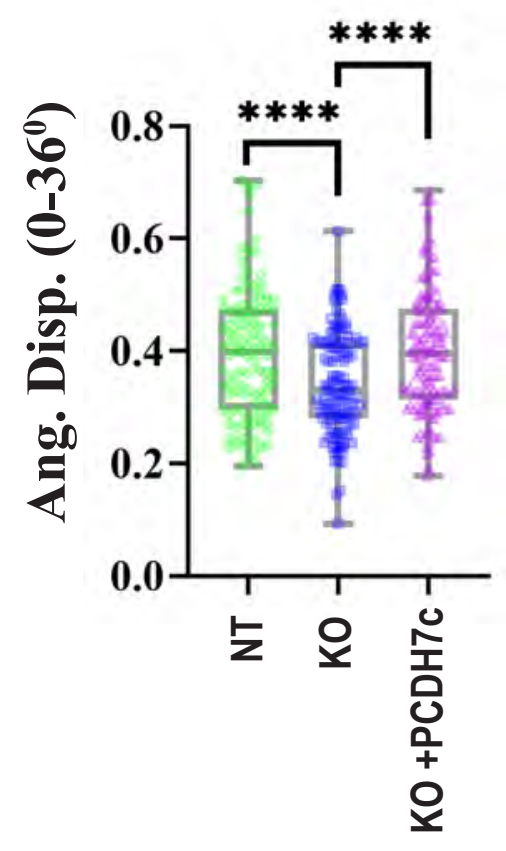

E

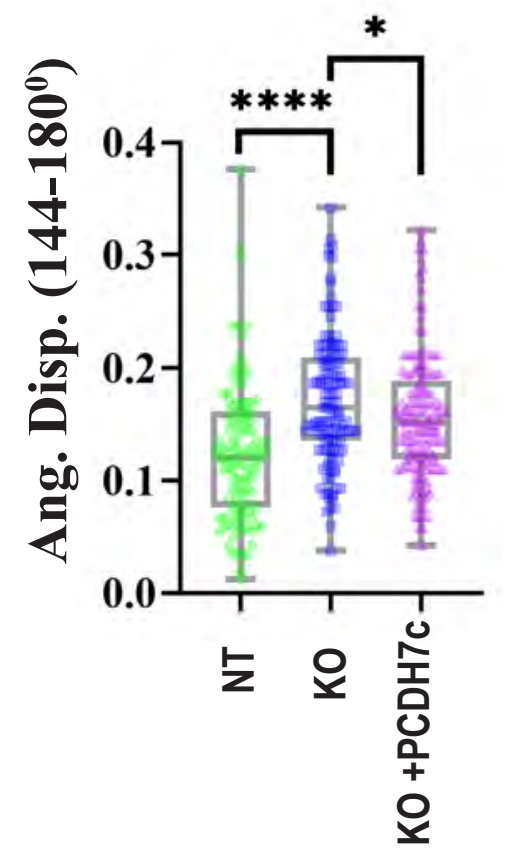

G
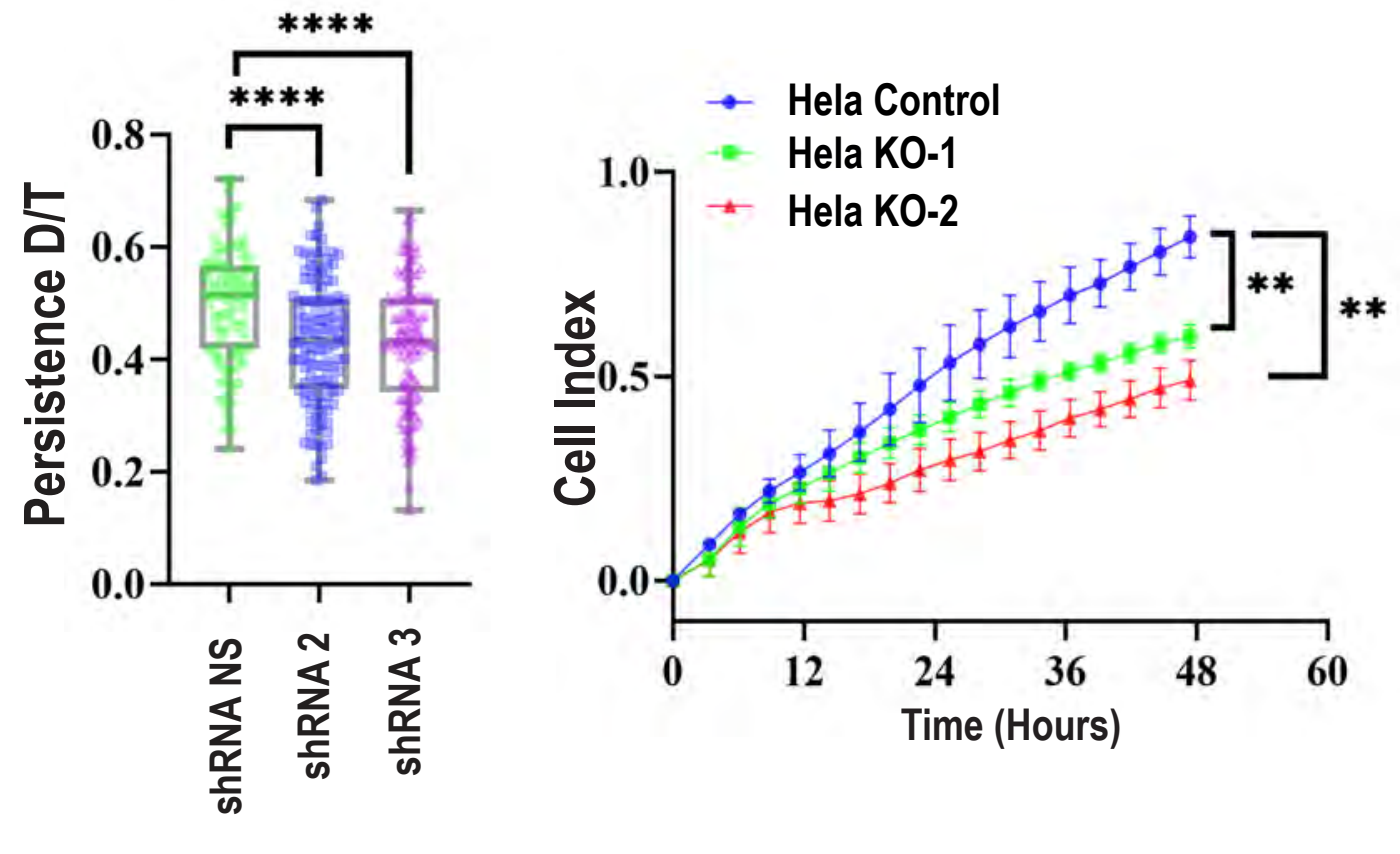
Qureshi et. al., Figure 3

온

A

솜

B

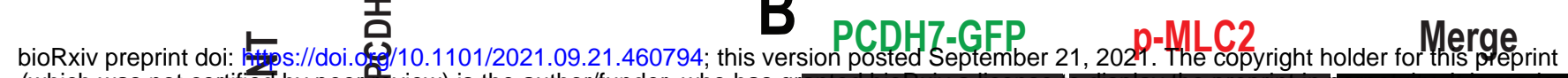
$190 \mathrm{KDa}$
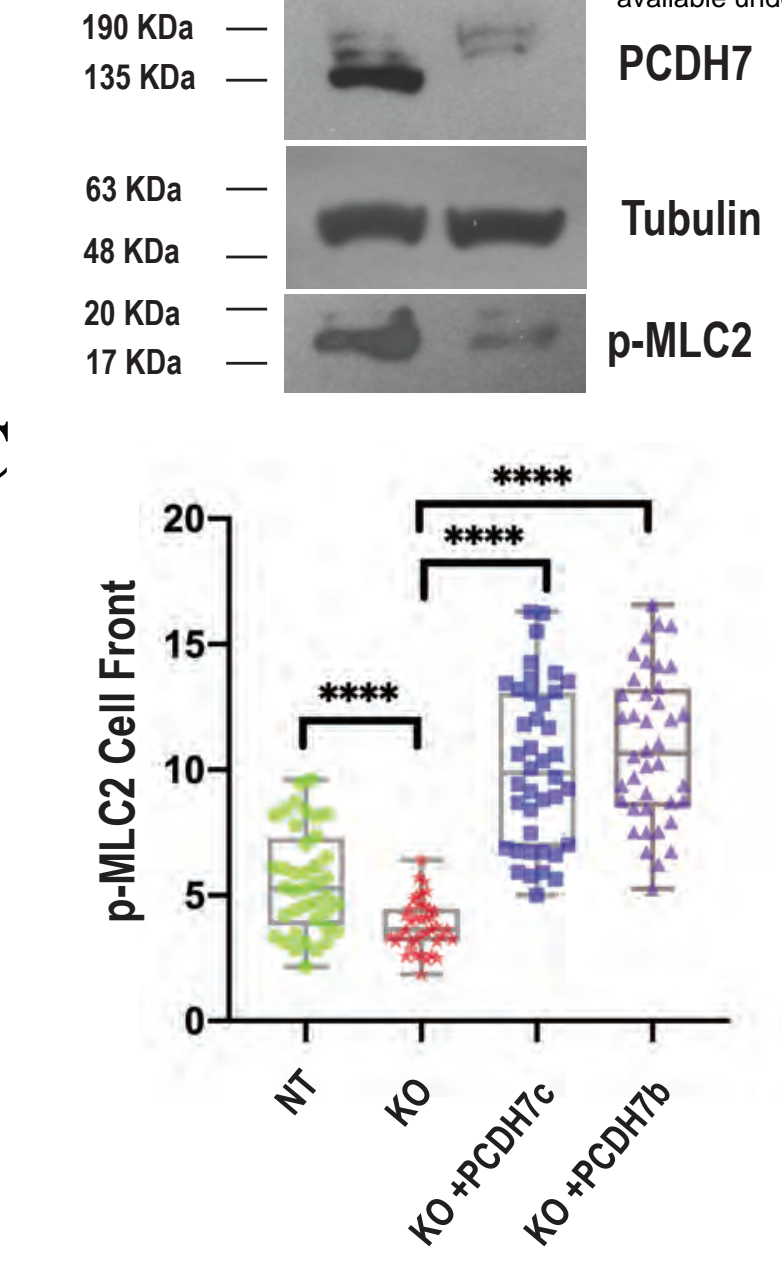

上
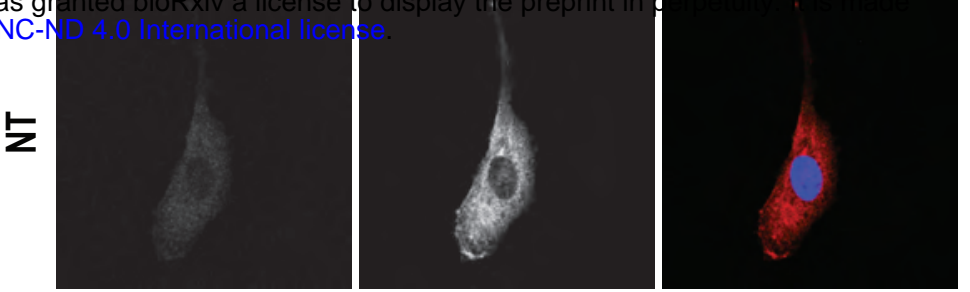

C

D
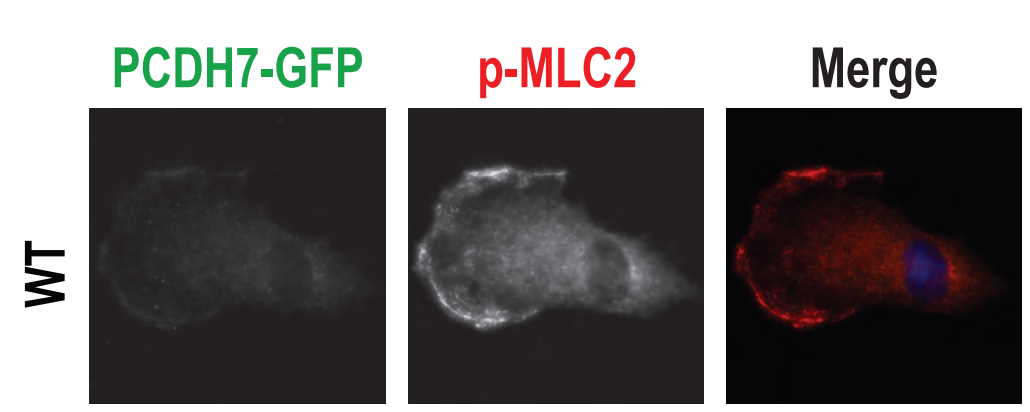

E
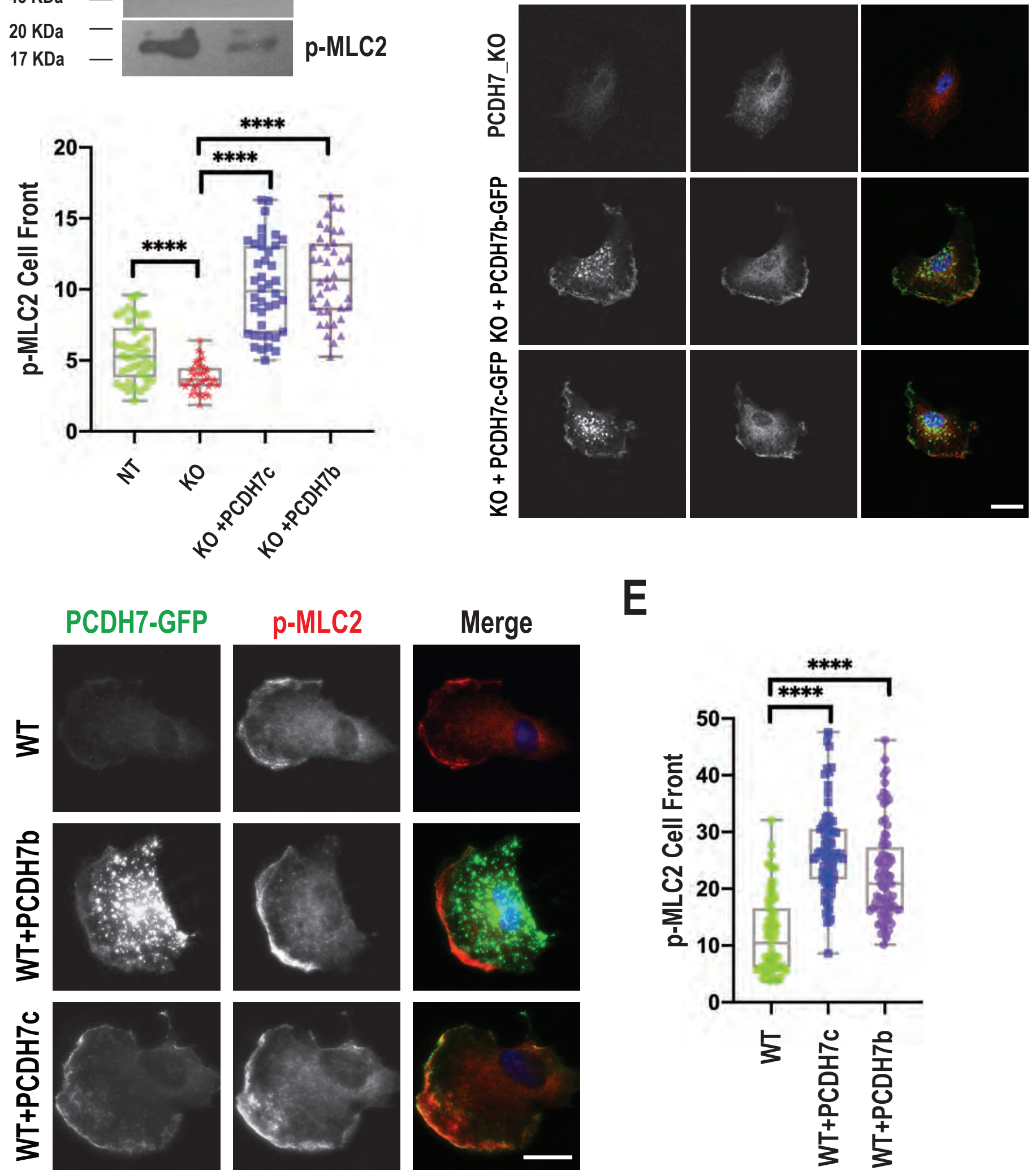
A

\section{CORO1B CFL2 GSOTEE}

MYH9

bioRxiv preprint doi: https://doi.org/10.1101/2021.09.21.460794; this version posted4SepPentrber 2ild 2021. The copycight holder for this preprint (which was not certified by peer review) is the author/funder, who has granted bioRxiv a license to displayithe preprint in perpetuity. It is made Eukaryotic translation initiation

Nuclear-transcribed mRNA catabolic
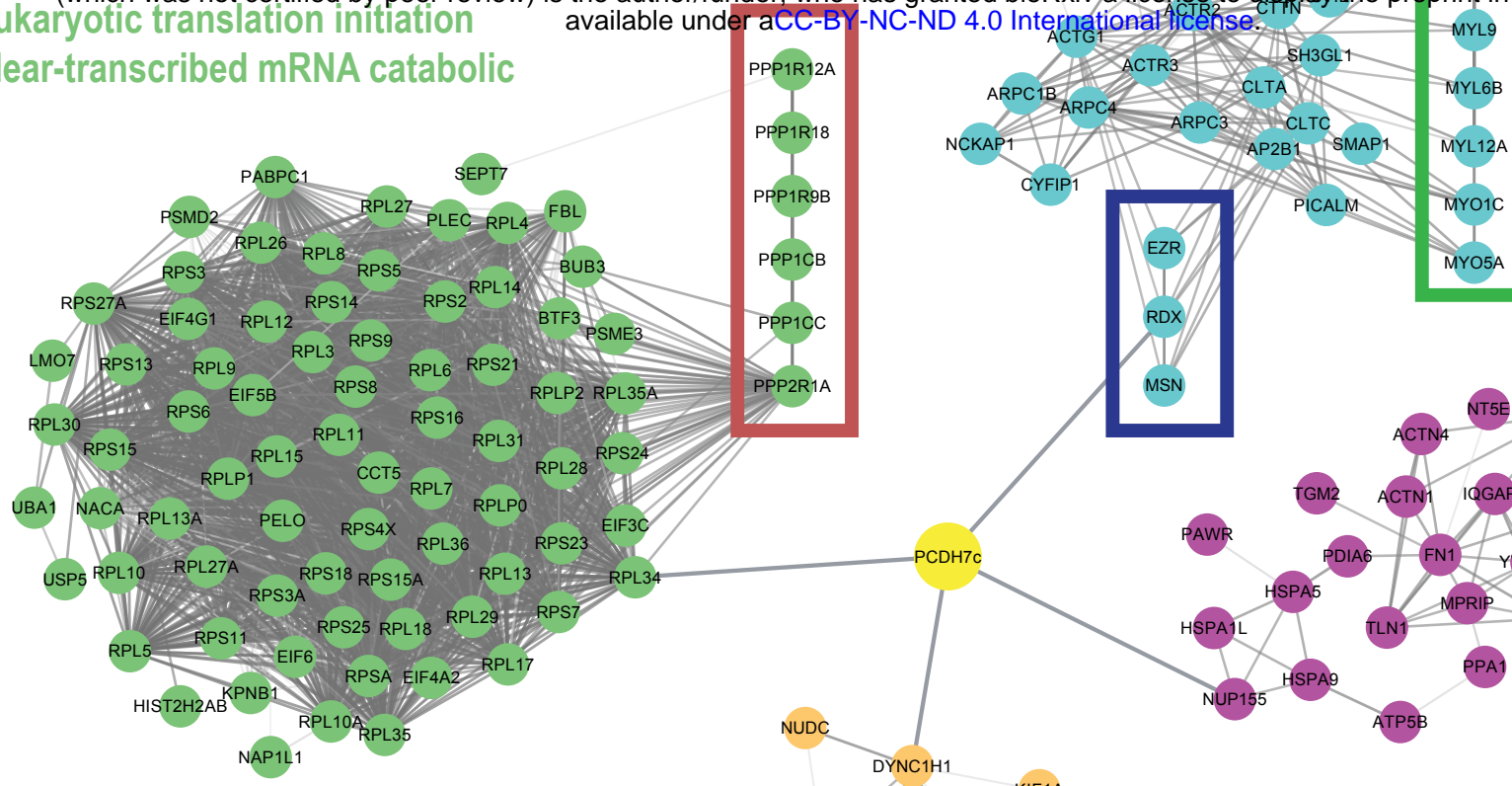
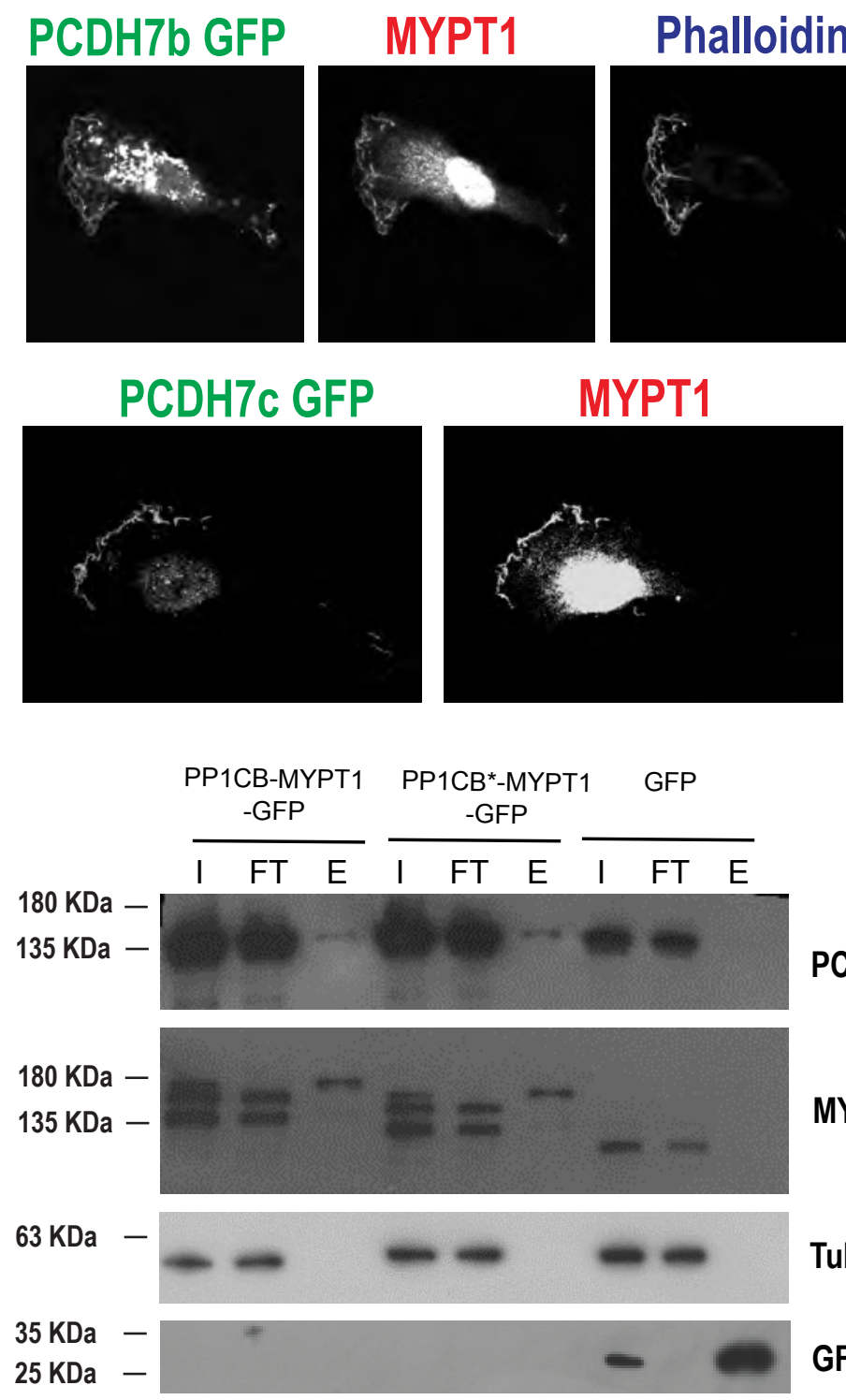
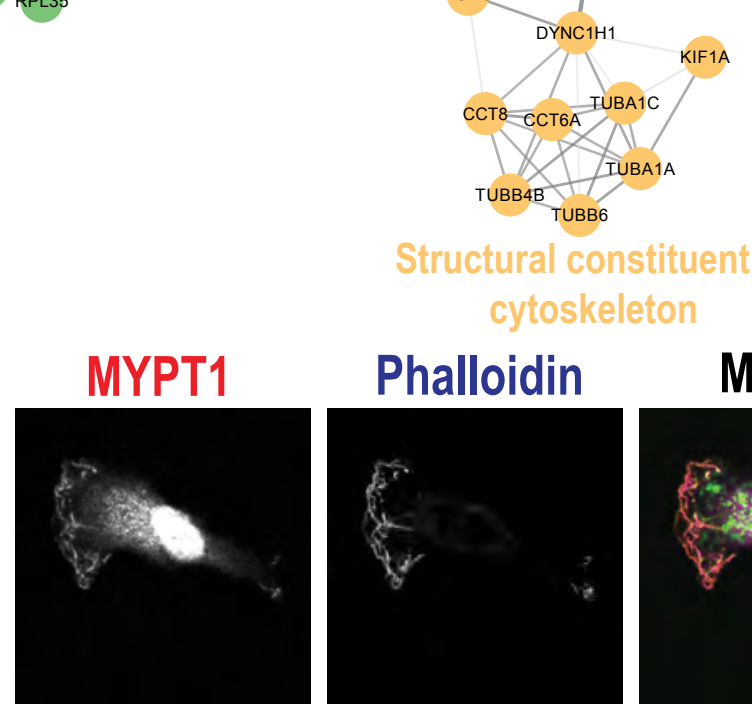

Structural constituent of

cytoskeleton

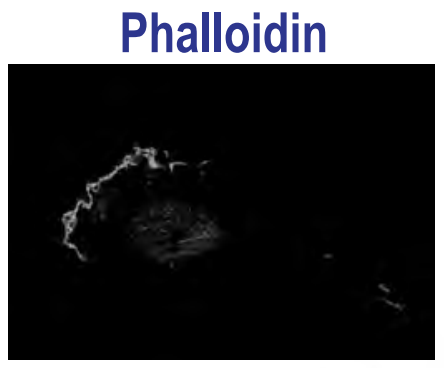

Phalloidin

D

PCDH7

MYPT1

Tubulin

GFP

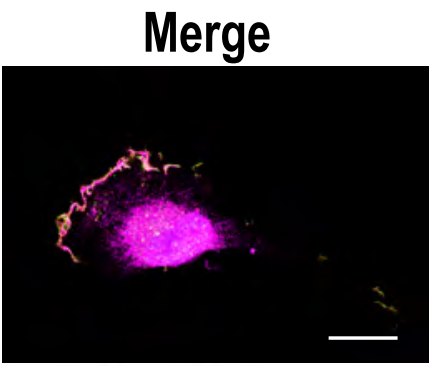

****

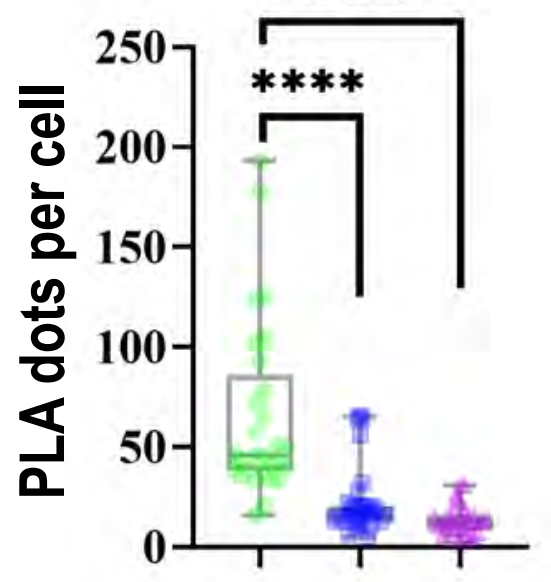

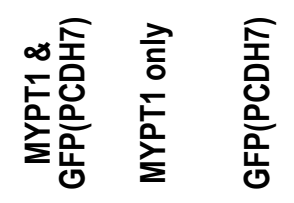




\section{Qureshi et. al., Figure 5}

A bioRxiv preprint doi: https://DAP/ $0.1101 / 2021.09 .21 .460794$ GF 5 Persion posted SeptembDAPUEG Fhe copyright holder for this preprint (which was not certified by neer review) is the author/funder who has aranted bioBxiva license to display the onrenrint in perpetuity. It is made
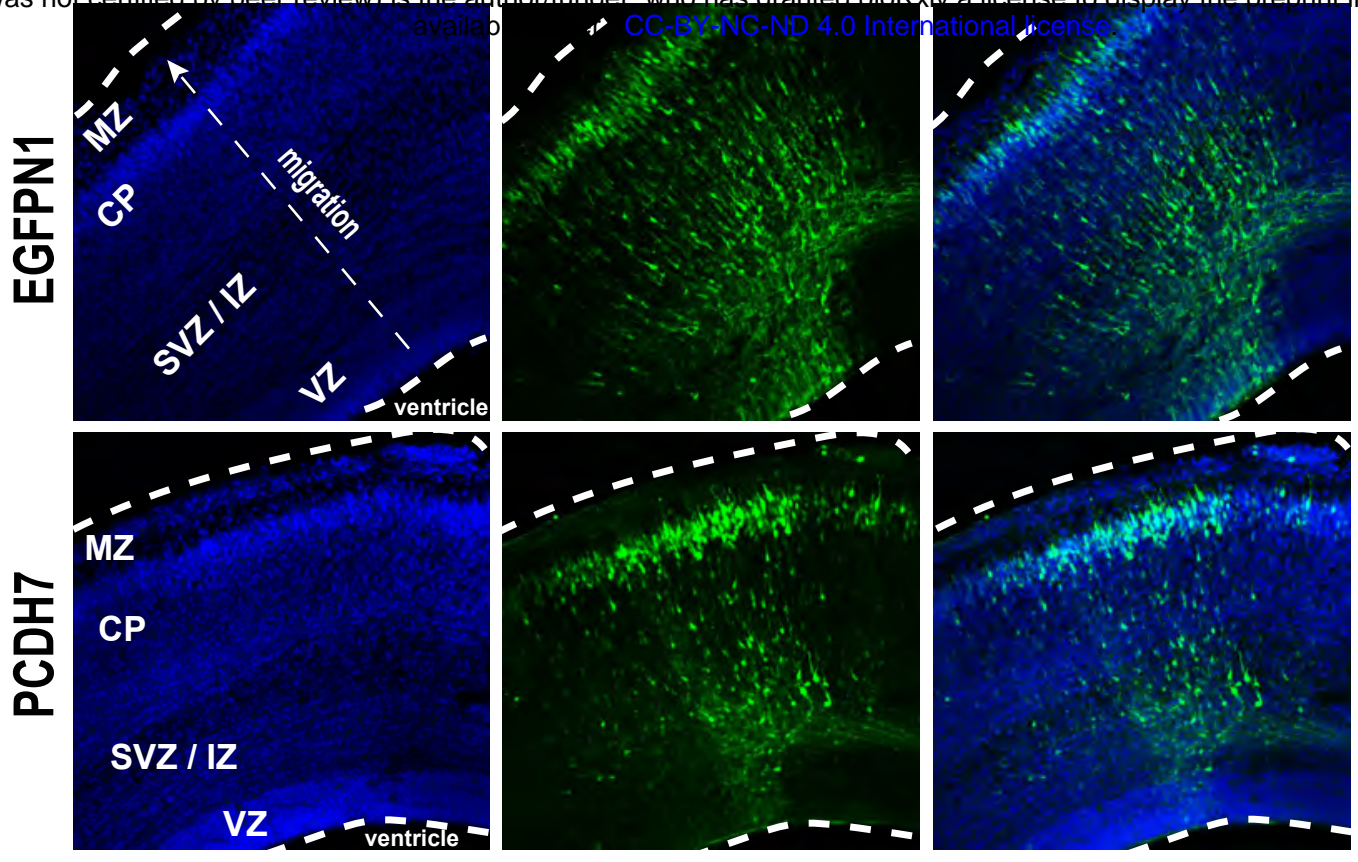

B

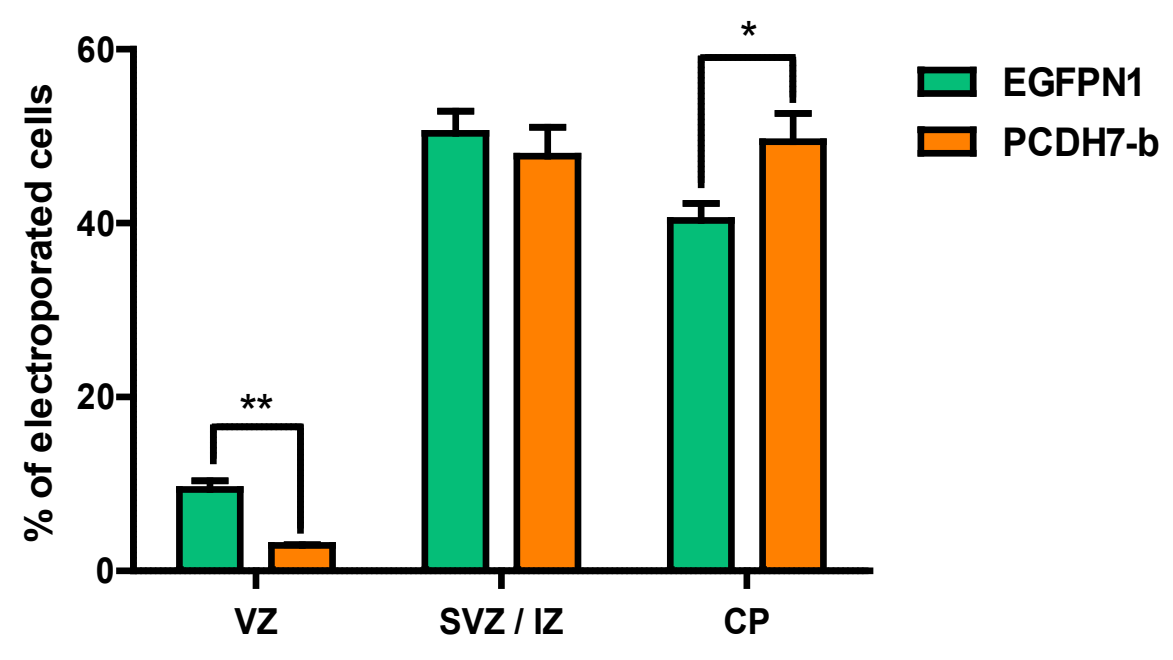


Qureshi et. al., Figure 6

A bioRxiv preprint doi: https://doi.org/10.1101/2021.09.21.460794; this version posted September 21, 2021. The copyright holder for this preprint

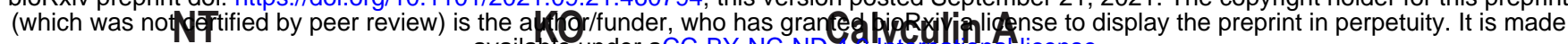
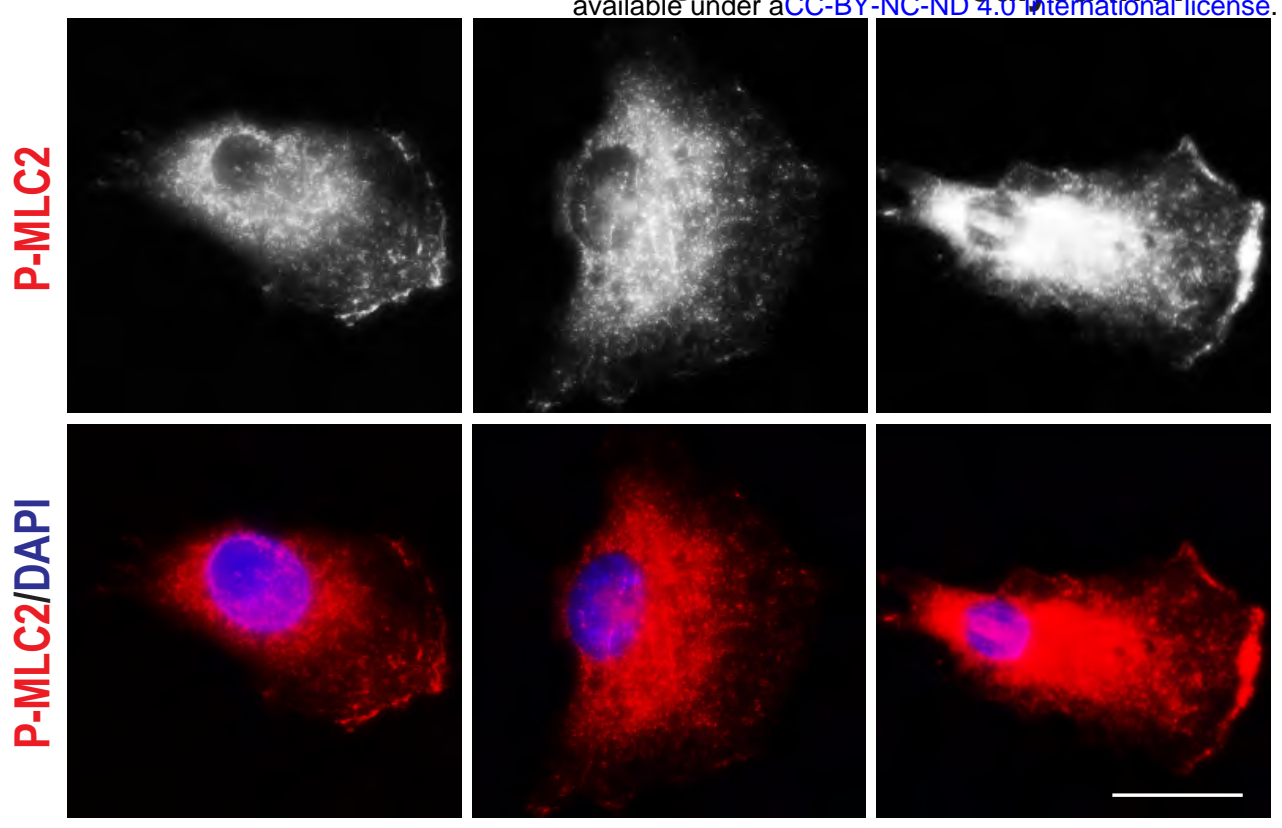

B
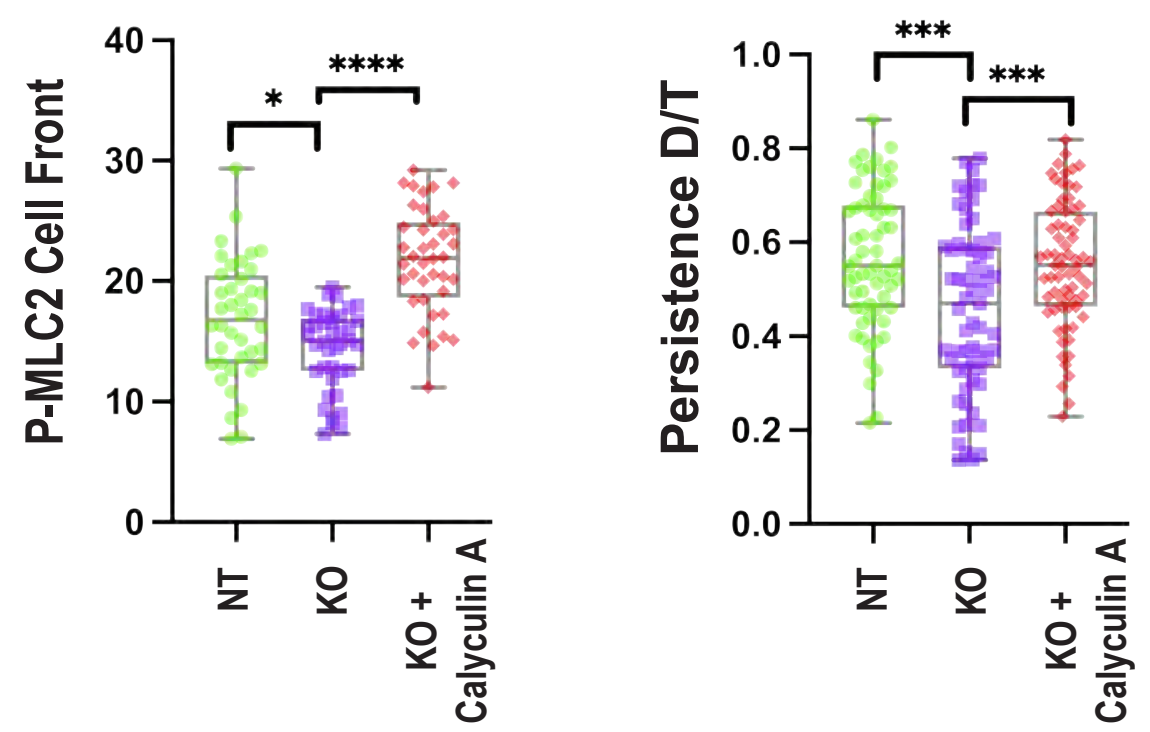